\title{
Coherent neutrino-nucleus scattering and new neutrino interactions
}

\section{Manfred Lindner, Werner Rodejohann and Xun-Jie Xu}

Max-Planck-Institut für Kernphysik, Postfach 103980, D-69029 Heidelberg, Germany

E-mail: manfred.lindner@mpi-hd.mpg.de, werner.rodejohann@mpi-hd.mpg.de, xunjie.xu@gmail.com

ABSTRACT: We investigate the potential to probe new neutrino physics with future experiments measuring coherent neutrino-nucleus scattering. Experiments with high statistics should become feasible soon and allow to constrain parameters with unprecedented precision. Using a benchmark setup for a future experiment probing reactor neutrinos, we study the sensitivity on neutrino non-standard interactions and new exotic neutral currents (scalar, tensor, etc). Compared to Fermi interaction, percent and permille level strengths of the new interactions can be probed, superseding for some observables the limits from future neutrino oscillation experiments by up to two orders of magnitude.

Keywords: Beyond Standard Model, Neutrino Physics

ARXIV EPRINT: 1612.04150 


\section{Contents}

1 Introduction 1

2 Coherent neutrino-nucleus scattering in the Standard Model 2

2.1 Cross section 2

2.2 Detection 3

3 Non-Standard Interactions in coherent $\nu-N$ scattering $\quad 6$

4 Exotic neutral currents in coherent $\nu-N$ scattering $\quad 8$

5 Sensitivities from a $\chi^{2}$-fit $\quad 14$

$\begin{array}{lll}5.1 & \text { Statistical treatment } & 14\end{array}$

5.2 Low energy determination of the Weinberg angle 16

$\begin{array}{lll}5.3 & \text { Non-Standard Interactions } & 16\end{array}$

$\begin{array}{ll}5.4 \text { Exotic neutral currents } & 17\end{array}$

$\begin{array}{llr}6 & \text { Conclusion } & 20\end{array}$

A Cross section calculation of coherent $\nu-N$ scattering in the Standard Model

B What if $N$ is a spin-1/2 or spin-1 particle?

C Relations of $\left(C_{a}, \bar{D}_{a}\right)$ with $\left(C_{a}^{(q)}, \bar{D}_{a}^{(q)}\right)$

\section{Introduction}

Coherent neutrino-nucleus scattering $(\mathrm{C} \nu N \mathrm{~S})$ [1-3] is a tree level process that is predicted by the Standard Model, but has not yet been observed. While being conceptually highly interesting and allowing measurements of electroweak observables at low momentum transfer, the process is also of phenomenological importance for future dark matter direct detection experiments [4]. Moreover, it holds the potential to probe new neutrino physics [5-8], which is the main focus of this paper.

In $\mathrm{C} \nu N \mathrm{~S}$, low energy neutrinos interact with the protons and neutrons in the nuclei coherently, which significantly enhances the cross section. While large fluxes of neutrinos are available from nuclear research or commercial reactors, the recoil energy of the nuclei is difficult to detect since it is very low. However, prompted partly by developments in dark matter direct detection experiments, modern low-threshold detectors make the detection of $\mathrm{C} \nu N \mathrm{~S}$ technically feasible $[9,10]$. Combined with smart shielding techniques, high-rate and low-background experiments are possible. ${ }^{1}$ Future $\mathrm{C} \nu N \mathrm{~S}$ experiments may thus provide

\footnotetext{
${ }^{1}$ See, for instance, refs. [11-15] for recent studies.
} 
precision test of neutrino interactions in the Standard Model and strong constraints on new physics related to neutrinos.

In this paper, we will study the sensitivities of $\mathrm{C} \nu N \mathrm{~S}$ on possible new neutrino interactions, mainly assuming Germanium detectors with sub-keV threshold, detecting reactor antineutrinos. For illustration, we will assume values of the experimental parameters within reach of current technology. ${ }^{2}$ To make our study applicable to various new physics models, we will adopt a model-independent approach, only considering the low energy effective operators of neutrinos and quarks. This includes not only the widely-discussed conventional Non-Standard Interactions (NSI) [16] which are in (chiral) vector form, but also more exotic interactions that could be in scalar or tensor form. What distinguishes this paper from previous studies of the potential implications of coherent scattering $[5-8,17]$, is the inclusion of such exotic interactions, and a comparative study on how different experimental details (such as energy threshold or neutrino flux uncertainty) influence the sensitivity on new physics.

The paper is organized as follows. We start by introducing $\mathrm{C} \nu N \mathrm{~S}$ in the Standard Model in section 2. Then we study the effect of new physics on $\mathrm{C} \nu N \mathrm{~S}$, based on effective operators of neutrinos and quarks, which can be divided into two cases, the conventional NSI in section 3 and exotic neutral currents in section 4 . In section 5 , we consider a benchmark setup for a $\mathrm{C} \nu N \mathrm{~S}$ experiment and perform $\chi^{2}$-fit on parameters from the Standard Model, NSI and exotic neutral currents to study the sensitivities of such an experiment on them. We conclude in section 6. Details on the calculation of the cross section with both spin-0 and spin- $1 / 2$ nuclei are delegated to appendix A and B. Some useful relations connecting the fundamental coupling constants of exotic neutral currents to the effective parameters in $\mathrm{C} \nu N \mathrm{~S}$ are given in appendix $\mathrm{C}$.

\section{Coherent neutrino-nucleus scattering in the Standard Model}

\subsection{Cross section}

In the Standard Model (SM), the Neutral Current (NC) interaction enables low energy neutrinos with $E_{\nu} \lesssim 50 \mathrm{MeV}$ (corresponding to length scales of $\gtrsim 10^{-14} \mathrm{~m}$ ) to interact coherently with protons and neutrons in a nucleus, which significantly enhances the cross section for a large nucleus. For a nucleus at rest with $Z$ protons and $N$ neutrons, the coherent cross section $[1,2,8]$ (see appendix A) is given by

$$
\frac{d \sigma}{d T}=\frac{\sigma_{0}^{\mathrm{SM}}}{M}\left(1-\frac{T}{T_{\max }}\right),
$$

where $\sigma_{0}^{\mathrm{SM}}$ is defined as

$$
\sigma_{0}^{\mathrm{SM}} \equiv \frac{G_{F}^{2}\left[N-\left(1-4 s_{W}^{2}\right) Z\right]^{2} F^{2}\left(q^{2}\right) M^{2}}{4 \pi}
$$

\footnotetext{
${ }^{2}$ See e.g. https://indico.mpp.mpg.de/event/3121/session/3/contribution/18/material/slides/0.pdf for details.
} 
Here $G_{F}, s_{W}=\sin \theta_{W}$, and $M$ are the Fermi constant, the Weinberg angle, and the mass of the nucleus, respectively. Since at low energies $s_{W}^{2} \approx 0.238$ [18], we have $N-\left(1-4 s_{W}^{2}\right) Z$ $\approx N-0.045 Z$, which implies that the cross section is dominated by the neutron number; $F\left(q^{2}\right)$ is the form factor of the nucleus and its coherent limit $\left(q^{2} \rightarrow 0\right)$ is 1 . For higher energies, due to loss of coherence, it will be smaller than 1 (for a recent quantitative study, see ref. [13]). The recoil energy $T$ of the nucleus has a maximal value $T_{\max }$, determined by the initial neutrino energy $E_{\nu}$ and the nucleus mass $M$ :

$$
T_{\max }\left(E_{\nu}\right)=\frac{2 E_{\nu}^{2}}{M+2 E_{\nu}} .
$$

For new physics beyond the SM, both eq. (2.1) and eq. (2.2) could be modified but eq. (2.3) still holds since it is determined purely from relativistic kinematics.

Eq. (2.2) was derived under the assumption that the nucleus is a spin-0 particle [1] (see also appendix $\mathrm{A}$ of this paper). However, this is not always true because a nucleus with odd $A=N+Z$ is a fermion, examples are ${ }^{73} \mathrm{Ge}$ or ${ }^{131} \mathrm{Xe}$. In appendix B, we calculate the simplest non-zero case, spin-1/2. It turns out that the difference is small, given by

$$
\left.\frac{d \sigma}{d T}\right|_{\operatorname{spin}=\frac{1}{2}}=\left.\frac{d \sigma}{d T}\right|_{\text {spin }=0}+\frac{\sigma_{0}^{\mathrm{SM}}}{M} \frac{T^{2}}{2 E_{\nu}^{2}} .
$$

Thus, the only difference is a term proportional to $T^{2} / E_{\nu}^{2}$, which is usually negligible in the coherence scattering process. In principle the nucleus could also be some higher spin particle but based on eq. (2.4) it is reasonable to deduce that the difference should be suppressed for a large nucleus.

\subsection{Detection}

Note that the recoil energy $T$ is the only measurable effect of coherent neutrino scattering. Depending on the type of detectors, the method to measure $T$ is very different. We will focus here on Germanium detectors which measure the ionization energy $I$, which is a fraction of the deposited recoil energy $T$. The fraction is defined as the quenching factor $Q=I / T$, typically within 0.15 to 0.3 for sub-keV recoil energies (see e.g. figures 5 and 7 in ref. [19]). The quenching factor at sub-keV energies is not well known due to lack of experimental data. In typical models like the one proposed by Lindhard et al. [20], the recoil energy depends on $Q$, so $I=T Q(T)$ would be a (not necessarily linear) function of $T$. However, no matter what the exact form of the function $I(T)$ would be, once $I$ is measured, it can be converted to $T$, provided that this function has been theoretically calculated [19] or experimentally measured. ${ }^{3}$ We assume that the quenching factor can be measured precisely in the future, and thus use the recoil energy $T$ rather than the ionization energy $I$. All the results in our paper can be simply converted from the $T$-dependence to the $I$-dependence, provided that the function $I(T)$ is determined.

\footnotetext{
${ }^{3}$ One approach to measure the quenching factor is to use neutron scattering, as performed in the CDEX-TEXONO collaboration above keV energies. For more details see https://wwwgerda.mpp.mpg.de/ symp/20_Ruan.pdf. In the future sub-keV measurements will be performed.
} 


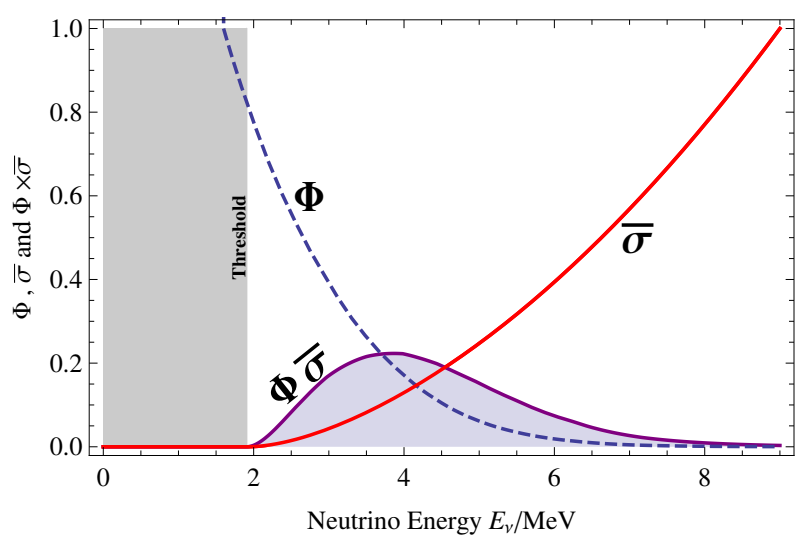

Figure 1. A typical reactor neutrino flux $\Phi$, reduced $\nu-N$ scattering cross section $\bar{\sigma}$ and their product. Units are arbitrary.

Generally for all types of detectors there is a detection threshold on $T$, denoted as $T_{\mathrm{th}}$. Therefore, for a given $E_{\nu}$ the recoil energy $T$ of detected events should be within the range $T_{\text {th }} \leq T \leq T_{\max }$ and the measurable reduced total cross section is

$$
\bar{\sigma}\left(T_{\mathrm{th}}, E_{\nu}\right) \equiv \int_{T_{\mathrm{th}}}^{T_{\max }} \frac{d \sigma}{d T} d T=\sigma_{0}^{\mathrm{SM}} \frac{\left(T_{\max }-T_{\mathrm{th}}\right)^{2}}{2 M T_{\max }} .
$$

Due to the threshold $T_{\text {th }}$, low energy neutrinos are impossible to detect if their energies are lower than

$$
E_{\nu, \mathrm{th}}=\frac{1}{2}\left(\sqrt{2 M T_{\mathrm{th}}+T_{\mathrm{th}}^{2}}+T_{\mathrm{th}}\right) \approx \sqrt{\frac{M}{2} T_{\mathrm{th}}} .
$$

For example, if $T_{\mathrm{th}}=0.1 \mathrm{keV}[12]$ then neutrinos should have $E_{\nu}>E_{\nu, \mathrm{th}} \approx 2 \mathrm{MeV}$ in order to be detected in a Ge detector. On the other hand, if we consider reactor neutrinos, the flux decreases exponentially at high energy. Therefore there is a limited range of $E_{\nu}$ for detection. To illustrate this, we plot in figure 1 the reduced cross section $\bar{\sigma}$ [given by eq. (2.5)], a typical reactor neutrino flux $\Phi$ and their product $\Phi \bar{\sigma}$, which is essentially proportional to the event rate. As figure 1 shows, the product $\Phi \bar{\sigma}$ is small at both low $(2 \mathrm{MeV})$ and high $(8 \mathrm{MeV})$ energies.

From the above discussion it is clear that the total event number decreases drastically when the detection threshold $T_{\mathrm{th}}$ is increased. To show this, we compute the total event numbers with different detection thresholds, plotted in figure 2, where one can see that the event number drops by 2 orders of magnitude if $T_{\text {th }}$ rises from $0.1 \mathrm{keV}$ to $0.8 \mathrm{keV}$. Therefore lowering the detection threshold is very crucial in order to obtain large event numbers. For this plot we have assumed a $100 \mathrm{~kg}$ Ge detector located $10 \mathrm{~m}$ away from a 1 GW (thermal power) reactor and taking data for five years. For the neutrino flux $\Phi\left(E_{\nu}\right)$, we have taken the spectrum from a recent theoretical calculation in ref. [21], normalized to $1.7 \times 10^{13} \mathrm{~cm}^{-2} \mathrm{~s}^{-1}$ (corresponding to $10 \mathrm{~m}$ distance from the reactor). Those values will serve as benchmark for our assumed future experiment, and can be used as a definition of our assumed "exposure" of

$$
\text { exposure }=5 \mathrm{~kg} \cdot \mathrm{yr} \cdot \mathrm{GW} \cdot \mathrm{m}^{-2} .
$$




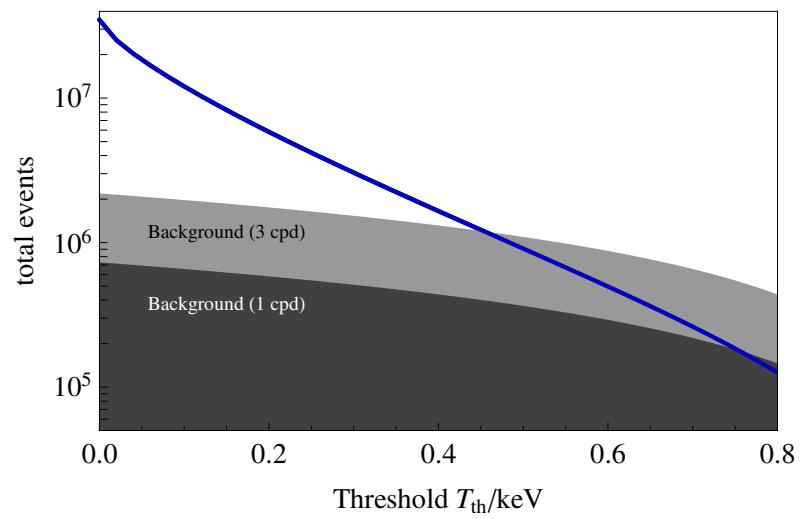

Figure 2. Total number of events compared with background $\left(1 \mathrm{cpd}=1\right.$ day $\left.^{-1} \mathrm{~kg}^{-1} \mathrm{keV}^{-1}\right)$. The total number decreases significantly when the detection threshold increases. We assume a $100 \mathrm{~kg}$ Ge detector located $10 \mathrm{~m}$ away from a reactor with $1 \mathrm{GW}$ thermal power, taking data for five years. For zero threshold, the total number of events is $3.8 \times 10^{7}$.

In figure 2 we also show the effect of an assumed constant background of $1 \mathrm{cpd}$ and $3 \mathrm{cpd}$ $\left(1 \mathrm{cpd}=1\right.$ day $\left.^{-1} \mathrm{~kg}^{-1} \mathrm{keV}^{-1}\right)$. The background may come from various sources, such as the intrinsic radioactivity of the material in the detector, ambient radioactivity near the nuclear reactor or cosmic rays. Estimation of the background is very much involved and depends significantly on the details of the detector. The GEMMA experiment [22] states a background level of about $2 \mathrm{cpd}$ and the TEXONO collaboration is aiming at developing a Ge detector with a background of $1 \mathrm{cpd}$ [10]. Note however that the mentioned background numbers apply to somewhat different energy scales and different background sources. Taking into account the low background levels that various double beta decay and dark matter direct detection exeriments have reached, plus noting the developements on active shielding at shallow depth [23], we estimate that such low background rates can be achieved.

In reality, not only the total event number but also the distribution of events will be measured, giving us a spectrum with respect to the recoil energy $T$. The spectrum provides more information than the total event number. The advantage to exploit the spectrum is that it is not influenced strongly by many uncertainties such as the flux normalization, the distance and fiducial mass of the detector, the form factor, etc. All those effects can be described roughly by an overall factor that enhances/reduces the total event number.

If the events are conservatively counted in many $T$-bins, the $i$-th bin with width $\Delta T$ starting from $T_{i}$, then the expectation of the event number $N_{i}$ in the $i$-th bin is

$$
N_{i}=\Delta t N_{\mathrm{Ge}} \frac{\sigma_{0}^{\mathrm{SM}}}{M} \int_{T_{i}}^{T_{i}+\Delta T} d T \int_{0}^{8 \mathrm{MeV}} d E_{\nu} \Phi\left(E_{\nu}\right) f^{\mathrm{SM}}\left(T, E_{\nu}\right) .
$$

Here $N_{\mathrm{Ge}}$ is the number of Ge nuclei ${ }^{4}$ in the detector and $\Delta t$ is the running time of detection, taken as 5 years. The neutrino flux $\Phi\left(E_{\nu}\right)$ has been taken from [21], and the

\footnotetext{
${ }^{4}$ Natural Germanium consists of ${ }^{70} \mathrm{Ge}(20.52 \%),{ }^{72} \mathrm{Ge}(27.45 \%),{ }^{73} \mathrm{Ge}(7.76 \%),{ }^{74} \mathrm{Ge}(36.52 \%)$ and ${ }^{76} \mathrm{Ge}$ (7.75\%). Here we take $A=72.6$ in average. Note that spin-dependent axial couplings in the Standard Model lead to smaller coherence factors depending on the spin of the nucleus, not on $N$ or $Z$ as the vector interaction that gives the leading contribution, see appendix B. This will be a permille effect, see [17].
} 


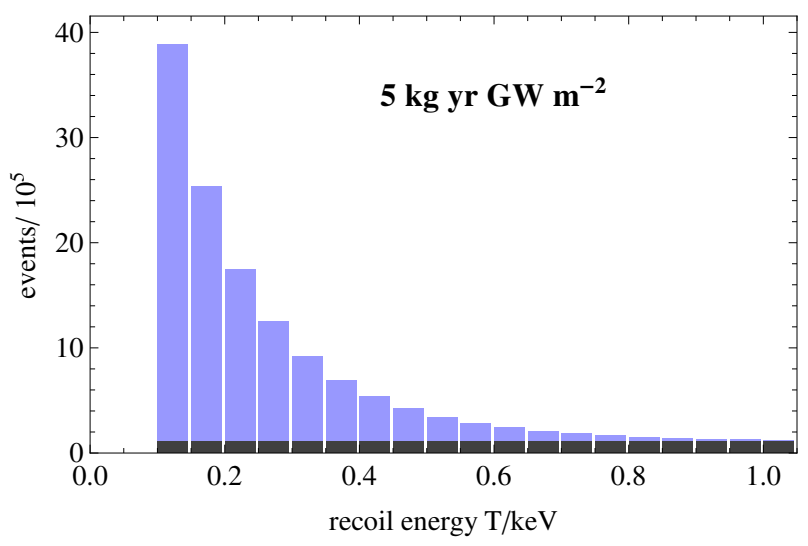

Figure 3. Expectation of event numbers in a $100 \mathrm{~kg}$ Ge detector running for 5 years, assuming a total flux of reactor neutrinos of $1.7 \times 10^{13} \mathrm{~cm}^{-2} \mathrm{~s}^{-1}$. The background (black) is assumed to be $3 \mathrm{cpd}$.

dimensionless function $f^{\mathrm{SM}}\left(T, E_{\nu}\right)$ is defined as [see eq. (2.1)]

$$
f^{\mathrm{SM}}\left(T, E_{\nu}\right)=\left\{\begin{array}{ll}
1-\frac{T}{T_{\max }\left(E_{\nu}\right)} & \text { for } T \leq T_{\max } \\
0 & \text { for } T>T_{\max }
\end{array} .\right.
$$

Note that when new physics beyond the SM is involved, one only needs to modify $\sigma_{0}^{\mathrm{SM}}$ in eq. (2.8) and $1-\frac{T}{T_{\max }\left(E_{\nu}\right)}$ in eq. (2.9) according to the new physics. Taking the flux from ref. [21] and setting the background at constant $3 \mathrm{cpd}$, the event numbers computed according to eq. (2.8) are presented in figure 3 as a function of $T$.

We should mention here that the calculation of the reactor neutrino flux is very complicated. Though a lot of effort was spent to calculate the flux in the literature (see e.g. [21, 24-27] and references therein), so far a very precise result is lacking, especially for neutrino energies below $2 \mathrm{MeV}$ where the error could be large as $7 \%$. The best understood range is from $2 \mathrm{MeV}$ to $6 \mathrm{MeV}$, but still with $3 \%$ error. Recently, measurements from the RENO [28, 29], Daya Bay [30] and Double Chooz [31] experiments showed disagreement with the theoretical calculation around $5 \mathrm{MeV}$, the infamous $5 \mathrm{MeV}$ bump. Its observation implies that we might have not fully understood the reactor neutrino flux. A particle physics origin of the bump seems very unlikely. In the next few years, both the theoretical understanding and experimental measurement will be significantly improved [32-34] so that the flux will be known more precisely and also the issue of the $5 \mathrm{MeV}$ bump will be resolved once our assumed future $\mathrm{C} \nu N \mathrm{~S}$ experiment is running. Anyway, the sensitivities of coherent $\nu-N$ scattering on new physics depend very little on the presence of the bump. A quantitative study on the influence of the $5 \mathrm{MeV}$ bump is presented below.

\section{Non-Standard Interactions in coherent $\nu-N$ scattering}

Coherent $\nu-N$ scattering could provide very strong constraints on neutrino Non-Standard Interactions (NSI). Those have been widely studied in the literature but so far the experimental constraints on some of its parameters are still very poor (see the reviews [16] and [35]), especially the couplings of neutrinos to quarks. 
In this work, only the neutrino-quark sector of NSI is relevant. The Lagrangian is

$$
\mathcal{L} \supset \frac{G_{F}}{\sqrt{2}} \sum_{q=u, d} \bar{\nu}_{\alpha} \gamma^{\mu}\left(1-\gamma^{5}\right) \nu_{\beta}\left[\varepsilon_{\alpha \beta}^{q V} \bar{q} \gamma^{\mu} q+\varepsilon_{\alpha \beta}^{q A} \bar{q} \gamma^{\mu} \gamma^{5} q\right]
$$

where $\alpha, \beta$ are the three flavors of neutrinos, and $\varepsilon_{\alpha \beta}^{q V}, \varepsilon_{\alpha \beta}^{q A}$ are the non-standard vector and axial-vector coupling constants, respectively. Interpreting the NSI terms in analogy to Fermi theory implies that the various $\varepsilon$ are given by

$$
\varepsilon \approx \frac{g_{X}^{2}}{g^{2}} \frac{M_{W}^{2}}{M_{X}^{2}},
$$

i.e. are related to new interactions mediated (for $\varepsilon \sim 0.1$ ) by $\mathrm{TeV}$-scale particles with mass $M_{X}$ ( $g_{X}$ denotes a new coupling constant). In neutrino oscillation experiments long-range forces have a similar effect as matter-induced NSIs [36]. We note that such light mediators could strongly affect the shape of the spectrum under study here, and thus distinguish both possibilities.

When the NSI Lagrangian (3.1) is added to the SM, the $\mathrm{C} \nu N \mathrm{~S}$ differential cross section is changed only by an overall factor. For the SM, the differential cross section is given in eq. (2.1) which is proportional to $\sigma_{0}^{\mathrm{SM}}$ given by eq. (2.2). For the NSI, following the calculation in appendix A, it is straightforward to obtain the result, which is simply replacing $\sigma_{0}^{\mathrm{SM}}$ with $\sigma_{0}^{\mathrm{NSI}}$, given by

$$
\sigma_{0}^{\mathrm{NSI}}=\frac{G_{F}^{2} Q_{\mathrm{NSI}}^{2} F^{2}\left(q^{2}\right) M^{2}}{4 \pi} .
$$

Here the modified weak charge $Q_{\text {NSI }}$ is defined as

$$
\begin{aligned}
Q_{\mathrm{NSI}}^{2} \equiv & 4\left[N\left(-\frac{1}{2}+\varepsilon_{e e}^{u V}+2 \varepsilon_{e e}^{d V}\right)+Z\left(\frac{1}{2}-2 s_{W}^{2}+2 \varepsilon_{e e}^{u V}+\varepsilon_{e e}^{d V}\right)\right]^{2} \\
& +4 \sum_{\alpha=\mu, \tau}\left[N\left(\varepsilon_{\alpha e}^{u V}+2 \varepsilon_{\alpha e}^{d V}\right)+Z\left(2 \varepsilon_{\alpha e}^{u V}+\varepsilon_{\alpha e}^{d V}\right)\right]^{2} .
\end{aligned}
$$

Setting the $\varepsilon$ to zero gives back the result from eq. (2.2). The axial vector couplings $\varepsilon_{\alpha \beta}^{q A}$ in eq. (3.1) do not appear in eq. (3.4) because of parity symmetry being present in large nuclei (see the discussion in appendix A). The cross section only depends on the vector couplings $\varepsilon_{\alpha \beta}^{q V}$, which for simplicity will be denoted by $\varepsilon_{\alpha \beta}^{q}$ henceforth. Even though this removes a lot of parameters, we are still confronted with a six-dimensional parameter space,

$$
\vec{\varepsilon} \equiv\left(\varepsilon_{e e}^{u}, \varepsilon_{e e}^{d}, \varepsilon_{\mu e}^{u}, \varepsilon_{\mu e}^{d}, \varepsilon_{\tau e}^{u}, \varepsilon_{\tau e}^{d}\right) .
$$

So far the best constraints [16] on $\varepsilon_{\alpha e}^{q}(\alpha=e, \tau)$ come from CHARM $\nu_{e}\left(\bar{\nu}_{e}\right) N$ inelastic scattering [37]. The $3 \sigma$-limits are

$$
\begin{aligned}
& -1.2<\varepsilon_{e e}^{u}<0.8, \\
& -0.7<\varepsilon_{e e}^{d}<1.4, \\
& -1.0<\varepsilon_{\tau e}^{u}<1.0, \\
& -1.0<\varepsilon_{\tau e}^{d}<1.0,
\end{aligned}
$$


assuming that for each bound only the corresponding coupling is non-zero. As one can see, these bounds are typically of order one. For the $\mu$ flavor, the best constraints are from $\mu^{-} \mathrm{Ti} \rightarrow e^{-} \mathrm{Ti}[16,38]$,

$$
\left|\varepsilon_{e \mu}^{u}\right|,\left|\varepsilon_{e \mu}^{d}\right|<1.4 \times 10^{-3},(3 \sigma) .
$$

This bound comes from a 1-loop diagram including the four fermion vertex of $\left|\varepsilon_{e \mu}^{q}\right|$. As a consequence, the result depends on the scale $\Lambda$ of the underlying UV complete model (recall that NSI in eq. (3.1) are non-renormalizable). In ref. [16] it is assumed $\ln \left(\Lambda / m_{W}\right) \approx 1$ to obtain this bound.

To understand how NSI affect $\mathrm{C} \nu N \mathrm{~S}$, we study the dependence of $\sigma_{0}^{\mathrm{NSI}}$ on the six parameters in eq. (3.5) with several plots in figure 4 . Each plot displays the ratio $\sigma_{0}^{\mathrm{NSI}} / \sigma_{0}^{\mathrm{SM}}$ as a function of two $\varepsilon$ in eq. (3.5), while the other four $\varepsilon$ are set to zero. Note that eq. (3.4) is symmetric under exchange of $\mu$ and $\tau$, thus we combine plots for $\varepsilon_{\mu e}^{q}$ and $\varepsilon_{\tau e}^{q}$ since for coherent $\nu-N$ scattering they have the same effect.

From the top two panels in figure 4 one can see that there is one direction (the green line) in which $\sigma_{0}^{\mathrm{NSI}} / \sigma_{0}^{\mathrm{SM}}$ is always equal to 1 , approximately at $\varepsilon_{\alpha e}^{u} \approx-\varepsilon_{\alpha e}^{d}$. Under the approximation that $N / Z \approx 1$ one can immediately derive this relation from eq. (3.4). It implies that $\mathrm{C} \nu N \mathrm{~S}$ does not have any sensitivity on NSI parameters along this direction, which has already been discussed in refs. [5, 9]. In the other panels, the direction with $\sigma_{0}^{\mathrm{NSI}} / \sigma_{0}^{\mathrm{SM}}=1$ also exists but in the form of a curve rather than a straight line. Therefore, degeneracies are present, which in case the NSI actually exist would need to be broken by other experiments, most notably neutrino oscillation experiments.

Figure 4 also shows that the ratio $\sigma_{0}^{\mathrm{NSI}} / \sigma_{0}^{\mathrm{SM}}$ could significantly deviate from 1 . Even for small values of $\varepsilon$ in the range $(-0.1,0.1), \sigma_{0}^{\text {NSI }}$ could vanish $\left(\sigma_{0}^{\mathrm{NSI}} / \sigma_{0}^{\mathrm{SM}}=0\right)$ or rise to twice the SM value $\left(\sigma_{0}^{\mathrm{NSI}} / \sigma_{0}^{\mathrm{SM}}=2\right)$. Therefore once coherent $\nu-N$ scattering is observed, it will provide a significant constraint on NSI parameters. Besides, among the six plots in figure 4 , only the top right one does not include $\sigma_{0}^{\mathrm{NSI}} / \sigma_{0}^{\mathrm{SM}}<1$, which implies that if the measured cross section is lower than the SM value, then $\varepsilon_{e e}^{u}$ or $\varepsilon_{e e}^{d}$ have to be non-zero in order to explain the deficit by NSI. A statistical analysis of the sensitivity on NSI will be performed in section 5 .

\section{Exotic neutral currents in coherent $\nu-N$ scattering}

Apart from the NSI which only couple neutrinos to quarks in (chiral) vector form, more "exotic" new interactions could be present. There are five types of possible interactions, scalar $(S)$, pseudo-scalar $(P)$, vector $(V)$, axial-vector $(A)$, and tensor $(T)$ interactions: ${ }^{5}$

$$
\mathcal{L} \supset \frac{G_{F}}{\sqrt{2}} \sum_{a=S, P, V, A, T} \bar{\nu} \Gamma^{a} \nu\left[\bar{q} \Gamma^{a}\left(C_{a}^{(q)}+\bar{D}_{a}^{(q)} i \gamma^{5}\right) q\right]
$$

\footnotetext{
${ }^{5}$ To make the following calculation more compact, we assume that the SM neutral current interaction is included in eq. (4.1) rather than adding eq. (4.1) to the SM Lagrangian. As a consequence, in the SM $C_{a}^{(q)}$ and $\bar{D}_{a}^{(q)}$ are non-zero.
} 

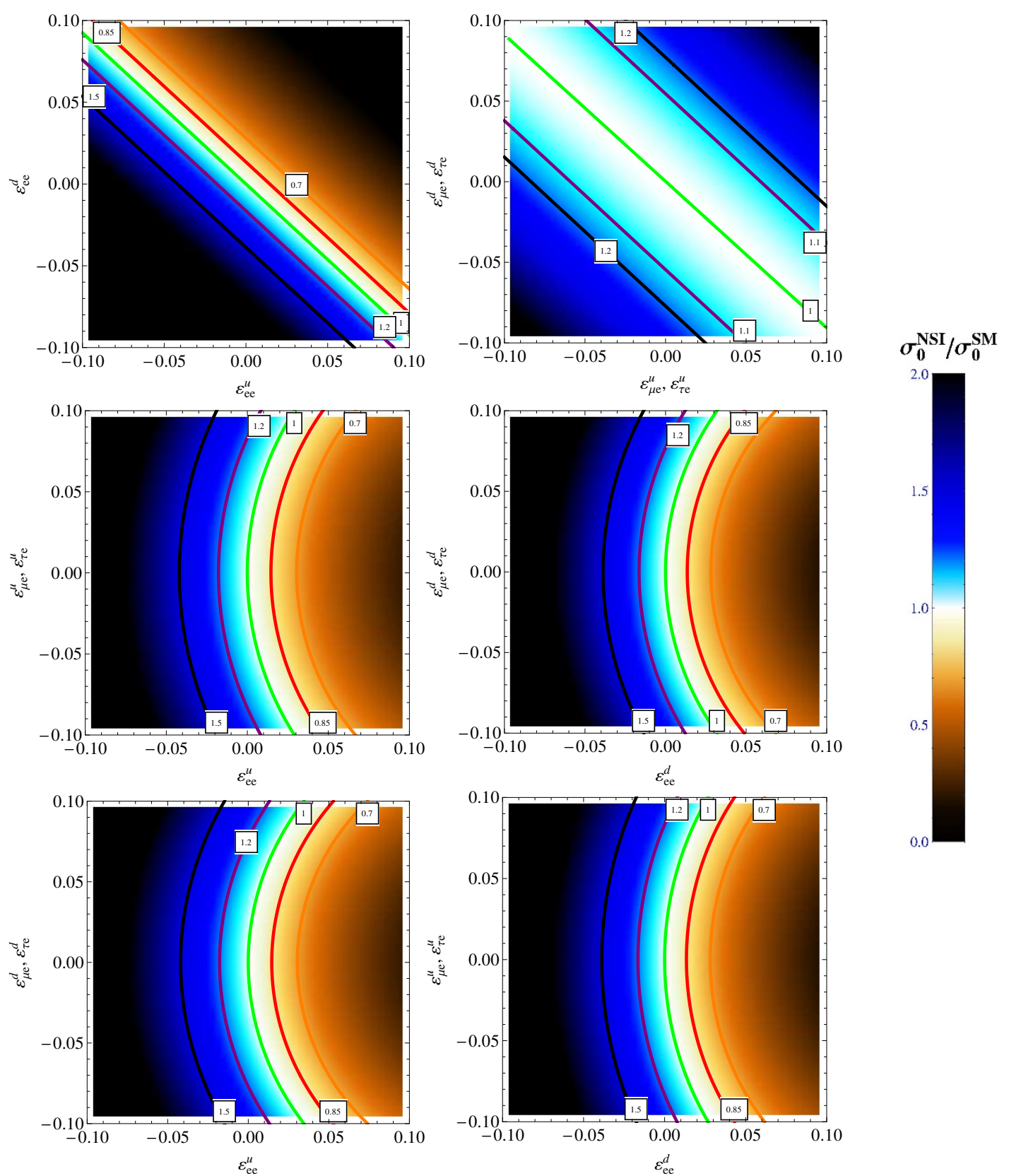

Figure 4. The effect of NSI parameters on the cross section ratio $\sigma_{0}^{\mathrm{NSI}} / \sigma_{0}^{\mathrm{SM}}$. The lower four plots are similar, because a large nucleus is almost symmetric with respect to $u \leftrightarrow d$.

where $q$ stands for $u$ and $d$ quarks and

$$
\Gamma^{a}=\left\{I, i \gamma^{5}, \gamma^{\mu}, \gamma^{\mu} \gamma^{5}, \sigma^{\mu \nu} \equiv \frac{i}{2}\left[\gamma^{\mu}, \gamma^{\nu}\right]\right\}
$$

In analogy to eq. (3.2), the $C_{a}^{(q)}$ and $\bar{D}_{a}^{(q)}$ are expected to be of order $\left(g_{X}^{2} / g^{2}\right)\left(M_{W}^{2} / M_{X}^{2}\right)$, with new exchange particles $M_{X}$ and coupling constants $g_{X}$. The coefficients $C_{a}^{(q)}$ and $\bar{D}_{a}^{(q)}$ in eq. (4.1) are dimensionless and in principle can be complex numbers. However if the inter- 
action term is not self-conjugate, it would be added by its complex conjugate, which is proportional to $\bar{\nu} \Gamma^{a} \nu\left[\bar{q} \Gamma^{a}\left(C_{a}^{(q) *}+\bar{D}_{a}^{(q) *} i \gamma^{5}\right) q\right]$ for $a=S, P, T$ and $\bar{\nu} \Gamma^{a} \nu\left[\bar{q} \Gamma^{a}\left(C_{a}^{(q) *}-\bar{D}_{a}^{(q) *} i \gamma^{5}\right) q\right]$ for $a=V, A$. Since $C_{a}^{(q) *}+C_{a}^{(q)}, \bar{D}_{a}^{(q)}+\bar{D}_{a}^{(q) *}$ and $i\left(\bar{D}_{a}^{(q)}-\bar{D}_{a}^{(q) *}\right)$ are real numbers, without loss of generality we can take $C_{a}^{(q)}$ and

$$
D_{a}^{(q)} \equiv \begin{cases}\bar{D}_{a}^{(q)} & (a=S, P, T) \\ i \bar{D}_{a}^{(q)} & (a=V, A)\end{cases}
$$

as real numbers. We will assume for simplicity that $C_{a}^{(u)}=C_{a}^{(d)}$ and $\bar{D}_{a}^{(u)}=\bar{D}_{a}^{(d)}$. This still leaves us with 10 free parameters.

A subtle issue related to $\sigma^{\mu \nu}$ and $\sigma^{\mu \nu} \gamma^{5}$ should be clarified here. When the tensor $\bar{\nu} \sigma^{\mu \nu} \nu$ is coupled to $\bar{q} \sigma^{\mu \nu} q$, there are two possibilities, $\bar{\nu} \sigma^{\mu \nu} \nu \bar{q} \sigma_{\mu \nu} q$ and $\epsilon^{\mu \nu \rho \sigma} \bar{\nu} \sigma_{\mu \nu} \nu \bar{q} \sigma_{\rho \sigma} q$. On the other hand, there could be new interactions such as $\bar{\nu} \sigma^{\mu \nu} \gamma^{5} \nu \bar{q} \sigma_{\mu \nu} q$ and $\bar{\nu} \sigma^{\mu \nu} \gamma^{5} \nu \bar{q} \sigma_{\mu \nu} \gamma^{5} q$, which seem not to be included in eq. (4.1). But due to the identity

$$
\sigma^{\mu \nu} i \gamma^{5}=-\frac{1}{2} \sigma_{\rho \sigma} \epsilon^{\mu \nu \rho \sigma}
$$

all these new possibilities can be transformed into the tensor form appearing in eq. (4.1):

$$
\bar{\nu} \sigma^{\mu \nu} \gamma^{5} \nu \bar{q} \sigma_{\mu \nu} q=\frac{i}{2} \epsilon^{\mu \nu \rho \sigma} \bar{\nu} \sigma_{\rho \sigma} \nu \bar{q} \sigma_{\mu \nu} \gamma^{5} q=\bar{\nu} \sigma^{\mu \nu} \nu \bar{q} \sigma_{\mu \nu} \gamma^{5} q
$$

Since the coherent nature of the scattering requires low energy, we can treat the nucleus in the coherent scattering as a point-like particle. Depending on the spin of the nucleus, it can be described by a scalar field, a Dirac field or even higher spin fields. As we have shown in eq. (2.4), for low energy scattering the difference of treating the nucleus as a spin-0 or spin- $1 / 2$ particle is negligible, and in fact identical to order $\left(T / E_{\nu}\right)^{2}$. In the following calculation we will treat the nucleus as a spin- $1 / 2$ particle since for automatic calculation implemented by packages (we use both FeynCalc [39, 40] and Package-X [41]) it is technically simpler than the scalar treatment. Consequently, the effective Lagrangian of neutrino-nucleus interactions has the same form as eq. (4.1) with $q$ replaced by the Dirac field $\psi_{N}$ of the nucleus, i.e.

$$
\mathcal{L} \supset \frac{G_{F}}{\sqrt{2}} \sum_{a=S, P, V, A, T} \bar{\nu} \Gamma^{a} \nu\left[\overline{\psi_{N}} \Gamma^{a}\left(C_{a}+\bar{D}_{a} i \gamma^{5}\right) \psi_{N}\right] .
$$

Note that to define the effective couplings of $\psi_{N}$ to $\nu$, here we use $\left(C_{a}, \bar{D}_{a}\right)$ which should be related to the more fundamental couplings $\left(C_{a}^{(q)}, \bar{D}_{a}^{(q)}\right)$. Since the relations are lengthy and also involve form factors, we present them in appendix C. From now on, we will consider $C_{a}$ and $\bar{D}_{a}$ as parameters of interest, and will present results in terms of those. We are not aware of literature limits on the parameters, which would have been obtained from past neutrino-nucleon scattering experiments. Since the event numbers in our benchmark experiment are much larger than in such experiments, the sensitivities we will derive later would surely be orders of magnitude better. 
From eq. (4.6), we can write down the scattering amplitude,

$$
i \mathcal{M}^{s^{\prime} s r^{\prime} r}=-i \frac{G_{F}}{\sqrt{2}} \bar{v}^{s}\left(p_{1}\right) P_{R} \Gamma^{a} v^{s}\left(k_{1}\right) \bar{u}^{r^{\prime}}\left(k_{2}\right) \Gamma^{a}\left(C_{a}+\bar{D}_{a} i \gamma^{5}\right) u^{r}\left(p_{2}\right) .
$$

Note that for general interactions, the coherent cross sections of $\nu N$ and $\bar{\nu} N$ are different [in the SM coherent $\nu N$ and $\bar{\nu} N$ cross sections are the same due to the approximate parity symmetry in nuclei, see comments after eq. (B.3)]. Since we are studying the coherent scattering of reactor neutrinos, only right-handed antineutrinos are considered. Therefore we have attached a $P_{R}=\left(1+\gamma^{5}\right) / 2$ projection to the initial neutrino state $\bar{v}^{s}\left(p_{1}\right)$, so that the trace technology applies,

$$
|\mathcal{M}|^{2}=\sum_{s s^{\prime}} \frac{1}{2} \sum_{r r^{\prime}}\left|\mathcal{M}^{s^{\prime} s r^{\prime} r}\right|^{2}
$$

The result is given by

$$
\begin{aligned}
\frac{d \sigma}{d T}= & \frac{G_{F}^{2} M}{4 \pi} N^{2}\left[\xi_{S}^{2} \frac{M T}{2 E_{\nu}^{2}}\right. \\
& +\xi_{V}^{2}\left(1-\frac{T}{T_{\max }}\right)-2 \xi_{V} \xi_{A} \frac{T}{E_{\nu}}+\xi_{A}^{2}\left(1-\frac{T}{T_{\max }}+\frac{M T}{E_{\nu}^{2}}\right) \\
& +\xi_{T}^{2}\left(1-\frac{T}{T_{\max }}+\frac{M T}{4 E_{\nu}^{2}}\right) \\
& \left.-R \frac{T}{E_{\nu}}+\mathcal{O}\left(\frac{T^{2}}{E_{\nu}^{2}}\right)\right]
\end{aligned}
$$

where

$$
\xi_{S}^{2}=\frac{1}{N^{2}}\left(C_{S}^{2}+D_{P}^{2}\right), \xi_{T}^{2}=\frac{8}{N^{2}}\left(C_{T}^{2}+D_{T}^{2}\right), \xi_{V}=\frac{1}{N}\left(C_{V}-D_{A}\right), \xi_{A}=\frac{1}{N}\left(C_{A}-D_{V}\right)
$$

and

$$
R \equiv \frac{2}{N^{2}}\left(C_{P} C_{T}-C_{S} C_{T}+D_{T} D_{P}-D_{T} D_{S}\right) .
$$

As we can see, the cross section only depends on 5 parameters, $\vec{\xi} \equiv\left(\xi_{S}, \xi_{V}, \xi_{A}, \xi_{T}, R\right)$, compared to the 10 parameters in eq. (4.6).

The first three lines of eq. (4.9) come from scalar and pseudo-scalar, vector and axial vector, and tensor interactions respectively while the $R$ term is an interference term of the (pseudo-) scalar and tensor interactions. Despite that $\xi_{S}$ contains both scalar and pseudoscalar contributions, for simplicity we will refer to $\xi_{S}^{2}$ as the scalar interaction of neutrinos with nuclei. In the same way, though the vector couplings $\left(C_{V}, D_{V}\right)$ and the axial vector couplings $\left(C_{A}, D_{A}\right)$ all appear in $\left(\xi_{V}, \xi_{A}\right)$, we still call $\xi_{V}^{2}$ and $\xi_{A}^{2}$ the vector and axial interactions, respectively.

Comparing eq. (4.9) to eq. (2.1), we obtain the SM values of these parameters,

$$
\vec{\xi}_{\mathrm{SM}} \equiv\left(0,1-\left(1-4 s_{W}^{2}\right) Z / N, 0,0,0\right) \approx(0,0.962,0,0,0),
$$

where the number 0.962 is computed for Germanium, i.e. by taking $N=40.6, Z=32$ and $s_{W}^{2}=0.238$. 


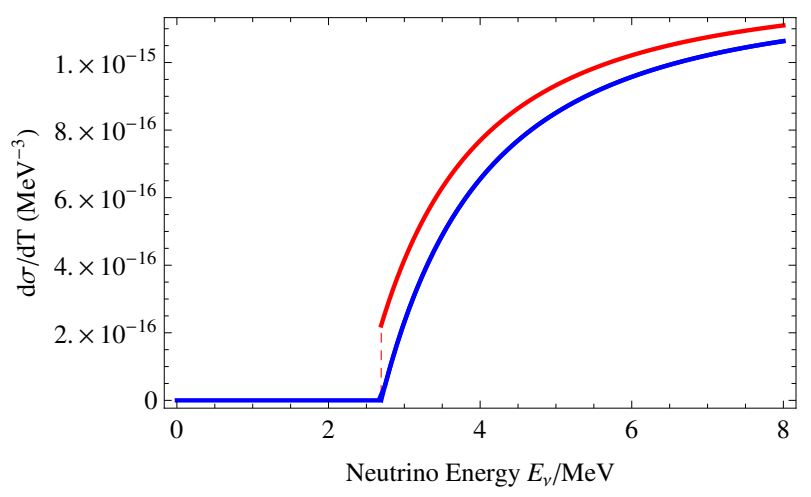

Figure 5. Effect of exotic neutral currents near the threshold. We plot $\frac{d \sigma}{d T}$ as a function of $E_{\nu}$ according to eq. (4.9) with fixed threshold $T_{\text {th }}=0.2 \mathrm{keV}$, corresponding to $E_{\nu} \gtrsim 2.7 \mathrm{MeV}$ for neutrinos. At $E_{\nu}=2.7 \mathrm{MeV}, T=T_{\max }$ and the cross section (2.1) in the SM vanishes. For exotic neutral currents the cross section (4.9) does not vanish for $T=T_{\max }$. The parameters are $\vec{\xi}=\vec{\xi}_{\text {SM }}$ for the blue curve and $\vec{\xi}=(0.4,1,0.1,0.1,0)$ for the red curve.

There are some noteworthy comments to make from eq. (4.9):

- There is no interference term of (axial) vector interactions with other interactions. But the vector interaction interferes with the axial interaction.

- The energy dependence of the $\xi_{V}^{2}$ term is the same as that in the SM [cf. eqs. (2.1) and (2.3)]. Hence, new vector interactions will not distort the recoil energy spectrum.

- The other terms (i.e. scalar, axial, tensor interaction terms and two interference terms) have different energy dependence. If any distortion on the recoil energy spectrum would be observed, then these new interactions could be the explanation.

- For vector interactions, $\frac{d \sigma}{d T}$ is zero at $T_{\max }\left(E_{\nu}\right)$ [defined in eq. (2.3)] but it could be non-zero if other types of interactions exist. This is shown in figure 5 where at the threshold the cross section is seen to be zero (blue curve) for the SM but non-zero (red curve) if other types of exotic neutral currents exist.

- Introducing exotic neutral currents (except for vector interactions) can not reduce the cross section since the sum of the other terms besides the $\xi_{V}^{2}$ term in eq. (4.9) is always above zero. So if the observed events are less than the expectation from the SM, one should consider modifications only limited to the vector sector rather than introducing scalar or tensor interactions.

The various modifications we have discussed so far (NSI, exotic neutral currents and the $5 \mathrm{MeV}$ bump) can influence the event numbers. In figure 6 we illustrate this for three examples. A feature of NSI is that they could result in a significant deficit (excess is also possible) of the event number, whereas exotic neutral currents only lead to an excess if $\xi_{V}$ is fixed at its SM value. In principle exotic neutral currents could also lead to a deficit by lowering $\xi_{V}$, but this is indistinguishable from the NSI case. The $5 \mathrm{MeV}$ bump in the neutrino flux also leads to an excess, but is not very significant. Here we take the size of 

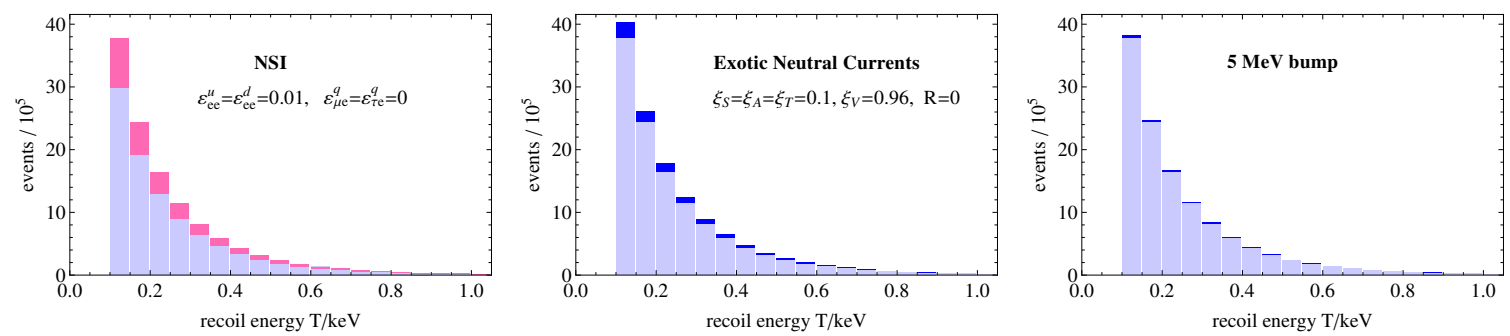

Figure 6. Event excess/deficit due to several possible modifications. The pink color is for deficit and dark blue for excess.

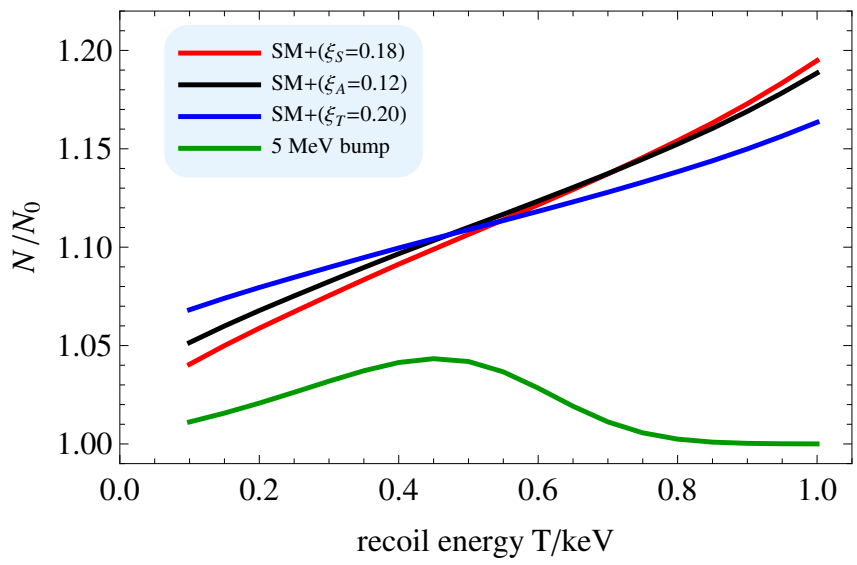

Figure 7. Distortion of the spectrum due to exotic neutral currents and the $5 \mathrm{MeV}$ bump. The red, black and blue curves correspond to scalar, axial vector and tensor interactions in addition to the SM. The green curve is produced by including the $5 \mathrm{MeV}$ bump in the neutrino flux, taken from ref. [34].

the $5 \mathrm{MeV}$ bump from a recent fit in ref. [34] (given by its figure 2). The excess in the 0.10$0.15 \mathrm{keV}$ bin is only about $1 \%$, which can be easily hidden in the systematic uncertainties. As mentioned before, since other experiments will collect with different reactor types a large amount of event numbers around the $5 \mathrm{MeV}$ bump, it is very likely that before a highly sensitive Ge detector with very small systematic uncertainties is running, the $5 \mathrm{MeV}$ bump problem will be solved (both in theory and experiment).

Another important difference is that the above three cases have very different effects on the distortion of the spectrum. NSI will not lead to any distortion at all since it only changes the overall factor in the differential cross section while the other two cases, exotic neutral currents and the $5 \mathrm{MeV}$ bump, lead to different distortions. In figure 7 we show variations of the event ratio $N / N_{0}$ as a function of $T$ in several situations, where $N_{0}$ is the event number expected from for the SM and $N$ includes new interactions or the $5 \mathrm{MeV}$ bump. For exotic neutral currents, we plot three examples to illustrate the effects from scalar, axial vector and tensor interactions with $\xi_{S}=0.18, \xi_{A}=0.12$ and $\xi_{T}=0.20$ respectively. All the other parameters, if not mentioned, have been set to the SM values given by eq. (4.12). As one can see, for exotic neutral currents the ratios increase with $T$ but the slopes are different. Scalar interactions would produce the strongest distortion on 
the spectrum followed by axial vector and then tensor. The $5 \mathrm{MeV}$ bump also generates an increasing ratio with respect to $T$ below $0.45 \mathrm{keV}$. However, the ratio drops down at higher energies and finally reaches 1 . The reason is that neutrinos at $5 \mathrm{MeV}$ will only contribute to the events below $0.7 \mathrm{keV}$ [cf. eq. (2.3)]. Thus in the range close to but less than $0.7 \mathrm{keV}$, the events from the $5 \mathrm{MeV}$ bump should quickly decrease. If all neutrinos in the bump only had energies exactly at $5 \mathrm{MeV}$, then the contribution should completely vanish above $0.7 \mathrm{keV}$. However, taking the width of the bump into consideration, the actual limit is a little higher than $0.7 \mathrm{keV}$.

\section{Sensitivities from a $\chi^{2}$-fit}

In this section, we will adopt $\chi^{2}$-fit to study the sensitivities of such our assumed future experiment. For convenience, let us state again our assumed exposure of $5 \mathrm{~kg} \cdot \mathrm{yr} \cdot \mathrm{GW} \cdot \mathrm{m}^{-2}$ from eq. (2.7), corresponding e.g. to a $100 \mathrm{~kg}$ Germanium detector running for 5 years, located at a distance of $10 \mathrm{~m}$ from a reactor with $1 \mathrm{GW}$ thermal power, normalized to a total flux of $1.7 \times 10^{13} \mathrm{~cm}^{-2} \mathrm{~s}^{-1}$. We will assume different thresholds of $T=0.1,0.2$ or $0.4 \mathrm{keV}$, and a constant background of $3 \mathrm{cpd}=3$ day $^{-1} \mathrm{~kg}^{-1} \mathrm{keV}^{-1}$.

\subsection{Statistical treatment}

Because the event number in each bin is very large, and thus almost in a Gaussian distribution, we can take the following $\chi^{2}$-function

$$
\chi^{2}(\xi, a)=\frac{a^{2}}{\sigma_{a}^{2}}+\sum_{T \text { bins }} \frac{\left[(1+a) N_{i}(\xi)-N_{i}^{0}\right]^{2}}{\sigma_{\text {stat }, i}^{2}+\sigma_{\text {sys }, i}^{2}},
$$

where $\xi$ denotes generally the parameters of interest, e.g. $\varepsilon_{\alpha \beta}^{q}$ for the NSI case or $\left(\xi_{S}, \xi_{V}\right.$, $\left.\xi_{A}, T, R\right)$ for exotic neutral currents. The event numbers in each bin as expected in the SM are denoted as $N_{i}^{0}$. The statistical uncertainty $\sigma_{\text {stat }, i}$ and the systematic uncertainty $\sigma_{\mathrm{sys}, i}$ of the event number in the $i$-th bin are given by

$$
\sigma_{\mathrm{stat}, i}=\sqrt{N_{i}+N_{\mathrm{bkg}, i}}, \sigma_{\mathrm{sys}, i}=\sigma_{f}\left(N_{i}+N_{\mathrm{bkg}, i}\right) .
$$

Here the background $N_{\mathrm{bkg}, i}$ is set at $3 \mathrm{cpd}\left(1 \mathrm{cpd}=1\right.$ day $\left.^{-1} \mathrm{~kg}^{-1} \mathrm{keV}^{-1}\right)$. We assume that $\sigma_{\mathrm{sys}, i}$ is proportional to the event number with a coefficient $\sigma_{f}$. Many systematic uncertainties simply change the total event number without leading to strong distortions of the spectrum, e.g. the uncertainties from the evaluation of the total flux of neutrinos, nuclear fuel supply, detection efficiency, fiducial mass of the detector, distance and geometry corrections, etc. To describe this part of systematic uncertainties, we introduce a normalization factor $a$ with a small uncertainty $\sigma_{a}$, while the other systematic uncertainties remain in $\sigma_{\mathrm{sys}, i}$. Of course in a more realistic study one should parametrize specifically the effect of every systematic uncertainty, some of which can not be described by this approach. For the current stage, we simply adopt eq. (5.1) for our sensitivity study, which nevertheless should provide realistic results. 


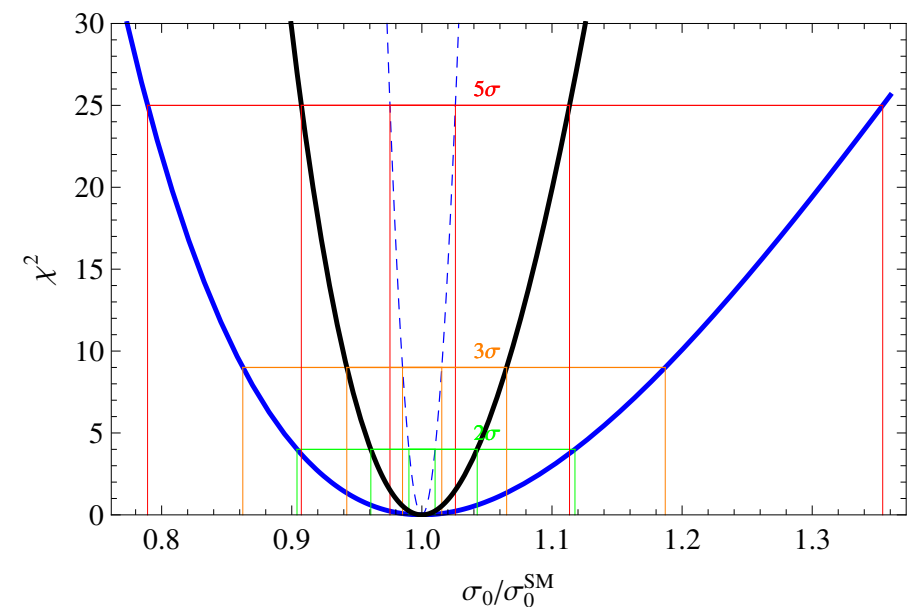

Figure 8. Sensitivity on the cross section ratio $\sigma_{0} / \sigma_{0}^{\mathrm{SM}}$. The blue solid, black solid and blue dashed curves are generated with a conservative configuration $\left(\sigma_{a}, \sigma_{f}, T_{\mathrm{th}}\right)=(5 \%, 3 \%, 0.4 \mathrm{keV})$, an intermediate configuration $\left(\sigma_{a}, \sigma_{f}, T_{\mathrm{th}}\right)=(2 \%, 1 \%, 0.2 \mathrm{keV})$ and an optimistic configuration $\left(\sigma_{a}, \sigma_{f}, T_{\mathrm{th}}\right)=(0.5 \%, 0.1 \%, 0.1 \mathrm{keV})$, respectively.

It is sometimes useful to know the value of $a$ at the minimum of $\chi^{2}$ analytically, which is

$$
a_{\min }=\frac{\sum_{i}\left(N_{i}^{0}-N_{i}\right) N_{i} /\left(\sigma_{\mathrm{stat}, i}^{2}+\sigma_{\mathrm{sys}, i}^{2}\right)}{\sigma_{a}^{-2}+\sum_{i} N_{i}^{2} /\left(\sigma_{\mathrm{stat}, i}^{2}+\sigma_{\mathrm{sys}, i}^{2}\right)} .
$$

One can use eq. (5.3) to marginalize $a$ and obtain the $\chi^{2}$-function that we are actually interested in,

$$
\chi^{2}(\xi) \equiv \chi^{2}\left(\xi, a_{\min }\right) .
$$

If coherent $\nu-N$ scattering has been successfully detected, the first task is to compare the measured total cross section $\sigma_{0}$ with the SM prediction $\sigma_{0}^{\mathrm{SM}}$ in eqs. $(2.1)$ and (2.2). The ratio $\sigma_{0} / \sigma_{0}^{\mathrm{SM}}$ indicates any deviation from the SM. One can compute the above $\chi^{2}$-function to estimate the sensitivity on this ratio ( $\xi$ in this case simply stands for $\sigma_{0}$ ). The result is shown in figure 8 , where we have assumed three different configurations:

(i) conservative configuration: $\left(\sigma_{a}, \sigma_{f}, T_{\mathrm{th}}\right)=(5 \%, 3 \%, 0.4 \mathrm{keV})$.

(ii) intermediate configuration: $\left(\sigma_{a}, \sigma_{f}, T_{\mathrm{th}}\right)=(2 \%, 1 \%, 0.2 \mathrm{keV})$.

(iii) optimistic configuration: $\left(\sigma_{a}, \sigma_{f}, T_{\mathrm{th}}\right)=(0.5 \%, 0.1 \%, 0.1 \mathrm{keV})$.

Even in the conservative configuration, the experiment can measure $\sigma_{0} / \sigma_{0}^{\mathrm{SM}}$ with good precision, $0.862<\sigma_{0} / \sigma_{0}^{\mathrm{SM}}<1.187$ at $3 \sigma$. In the intermediate case, $0.942<\sigma_{0} / \sigma_{0}^{\mathrm{SM}}<1.065$, while for the optimistic case $0.985<\sigma_{0} / \sigma_{0}^{\mathrm{SM}}<1.015$, all at $3 \sigma$. As it turns out, the improvement in sensitivity on new physics parameters between the conservative and intermediate configuration is about a factor of two. Roughly another factor of two can be gained when going from the intermediate configuration to the somewhat overly optimistic one. The choices we made for the various configurations should therefore give a feeling on the final sensitivity of such experiments. 


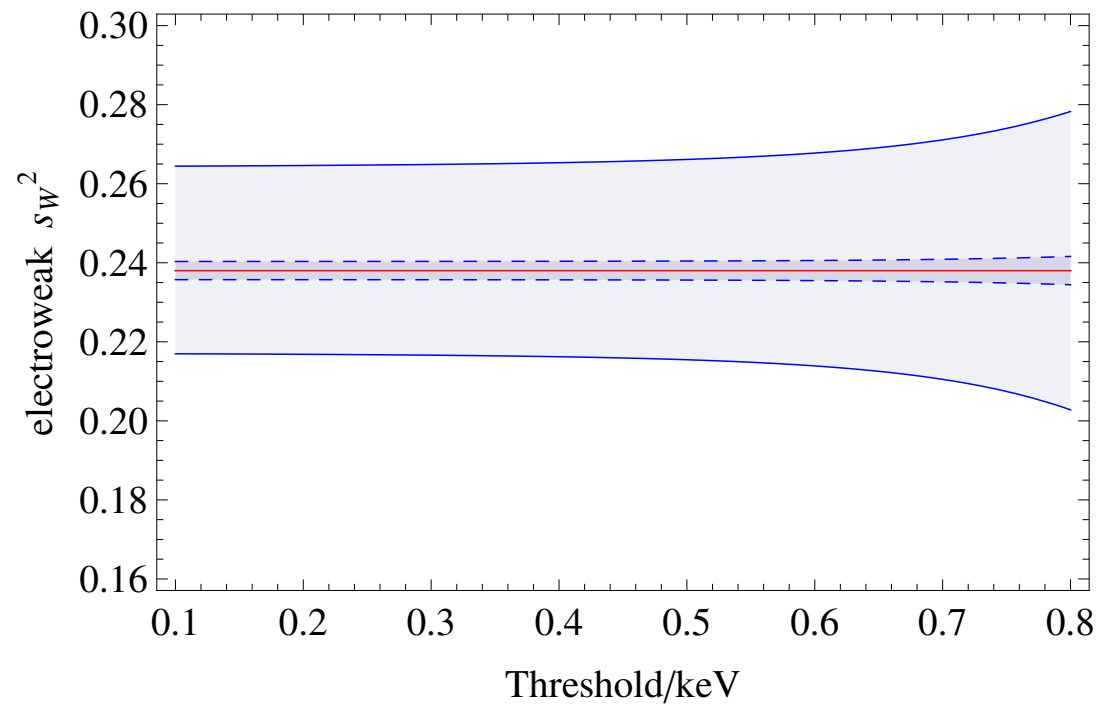

Figure 9. Sensitivity on the electroweak mixing angle $\sin ^{2} \theta_{W}$. The central value (red line) is the literature value of 0.238 and the blue solid and dashed curves denote $3 \sigma$-bounds in the conservative and optimistic configuration, respectively.

\subsection{Low energy determination of the Weinberg angle}

The measurement of $\sigma_{0}$ can also be converted into a measurement of the electroweak angle $\sin ^{2} \theta_{W}$ according to eq. (2.2), which would provide important complementary insight into electroweak precision observables at low energies. In figure 9 we show the sensitivity of this experiment on $\sin ^{2} \theta_{W}$, assuming its SM value at low scale of 0.238 (red line). The blue curves represent $3 \sigma$-bounds, solid for a conservative configuration $\left(\sigma_{a}, \sigma_{f}\right)=(5 \%, 3 \%)$ and dashed for a optimistic one $\left(\sigma_{a}, \sigma_{f}\right)=(0.5 \%, 0.1 \%)$. From figure 9 we can see that in the conservative configuration $\sin ^{2} \theta_{W}$ is expected to be measured, depending on the threshold, to a good precision between $10 \%$ and $20 \%$, while in the optimistic configuration, this would be improved roughly by an order of magnitude. For a threshold of $0.1 \mathrm{keV}$, the precision at $3 \sigma$ is \pm 0.0022 , or about $1 \%$, to be compared with the dedicated P2 experiment [42], which aims at a $1 \sigma$ precision of $0.13 \%$.

\subsection{Non-Standard Interactions}

The effect of the conventional NSI, as we have discussed in section 3, is merely a correction on the overall factor $\sigma_{0}^{\mathrm{SM}}$. The dependence of $\sigma_{0}^{\mathrm{NSI}}$ on various $\varepsilon$ parameters has been studied in section 3 and was displayed in figure 4 . We will study here the sensitivities of each $\varepsilon$ individually, assuming that all others are zero. The sensitivities on the NSI parameters in both the conservative and optimistic configurations are presented in figure 10. The left panel is for $\varepsilon_{e e}^{q}$ with $q=u$ or $d$. The right panel is for $\varepsilon_{\alpha e}^{q}$ with $\alpha=\mu$ or $\tau$, the cases are indistinguishable. The left panel of figure 10 shows that the $\mathrm{C} \nu N \mathrm{~S}$ experiment in the conservative configuration could constrain $\varepsilon_{e e}^{q}$ to order $10^{-2}$, much better than the current best bounds given in eqs. (3.6) and (3.7), which are typically of order 1. If one takes the optimistic configuration, then the constraint would reach the order of $10^{-3}$. The constraints 

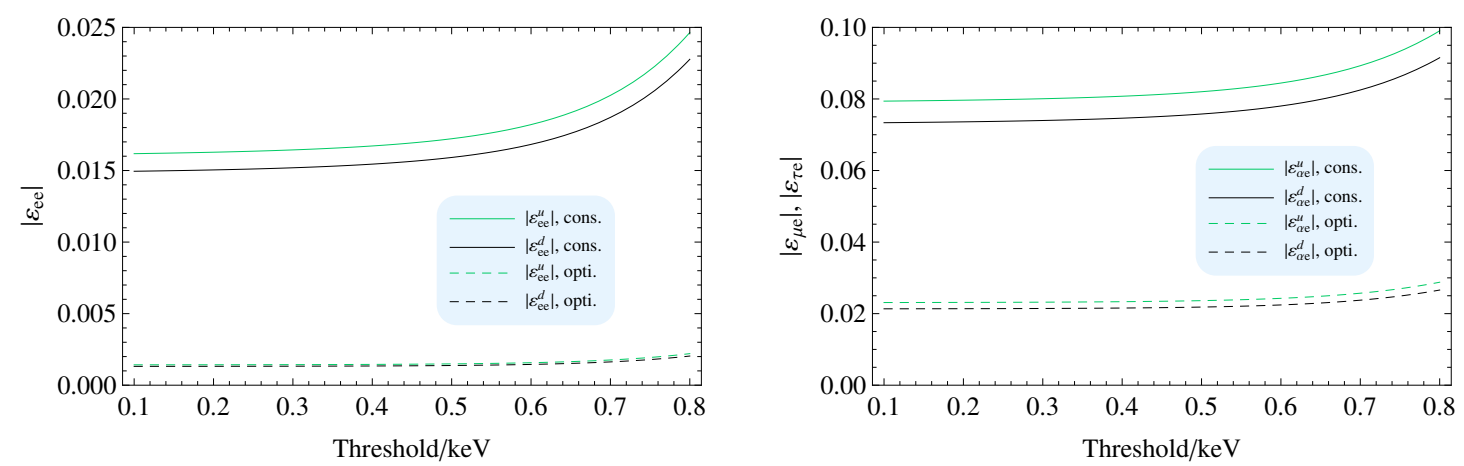

Figure 10. Sensitivities on the conventional NSI parameters $(3 \sigma)$. The solid and dashed curves are generated for a conservative and optimistic configuration, respectively.

on $\varepsilon_{\mu e}^{q}$ and $\varepsilon_{\tau e}^{q}$, however, are relatively weaker, about 0.07 to 0.10 (0.02 to 0.03$)$ for the conservative (optimistic) configuration. This can be easily understood from the form of $Q_{\text {NSI }}$ in eq. (3.4). For the $\tau$-channel, this is still a significant improvement compared to the current bound in eqs. (3.8) and (3.9) while for the $\mu$-channel, the current known bound is already very strong [see eq. (3.10)]; therefore, even if we take the optimistic estimation, the constraint would not exceed the known bound.

To summarize the comparison discussed above, we plot those bounds in figure 11 . The blue and dark blue bars shows the $3 \sigma$ bounds from our assumed $\mathrm{C} \nu N \mathrm{~S}$ experiment with conservative and optimistic configuration, respectively. The light blue bars represent the best known bounds from the review [16], see eqs. (3.6) and (3.10). We also add the expected bounds [43] from the future long-baseline neutrino experiment DUNE in the plot. The sensitivity of DUNE on NSI is based on the modified matter effect of neutrino oscillations caused by NSI parameters. The parameter set constrained by DUNE is actually

$$
\varepsilon_{\alpha \beta} \equiv \sum_{f=u, d, e} \varepsilon_{\alpha \beta}^{q} \frac{n_{f}}{n_{e}} \approx 3 \varepsilon_{\alpha \beta}^{u}+3 \varepsilon_{\alpha \beta}^{d}+\varepsilon_{\alpha \beta}^{e},
$$

where $n_{f}$ is the number density of the corresponding fermion $f$. Their relative density ratio $\left(n_{u}: n_{d}: n_{e}\right)$ is approximately $(3: 3: 1)$ in the Earth crust. Focusing on one parameter at a time, the limits on $\varepsilon_{\alpha \beta}$ from ref. [43] can be translated into limits on $\varepsilon_{\alpha \beta}^{q}$. This serves to compare the sensitivities and is displayed in figure 11. Even the conservative configuration improves the bounds on $\varepsilon_{\tau e}^{u, d}$ and $\varepsilon_{e e}^{u, d}$ considerably beyond current limits and future DUNE sensitivities. The limits obtainable in our benchmark experiment are summarized in table 1.

\subsection{Exotic neutral currents}

Next we shall study the sensitivity on the exotic neutral currents discussed in section 4 . The cross section (4.9) only depends on 5 effective parameters $\left(\xi_{S}, \xi_{V}, \xi_{A}, \xi_{T}, R\right)$, and we will perform a $\chi^{2}$-fit on those. Similar to the NSI analysis, we will focus on one type of exotic interactions at a time. However, in our parametrization $\xi_{V}$ is necessarily non-zero as it includes the SM contribution. So each time we take two non-zero parameters in the fit. One is $\xi_{V}$ and the other one is from exotic couplings. We also take $R$ to zero since 


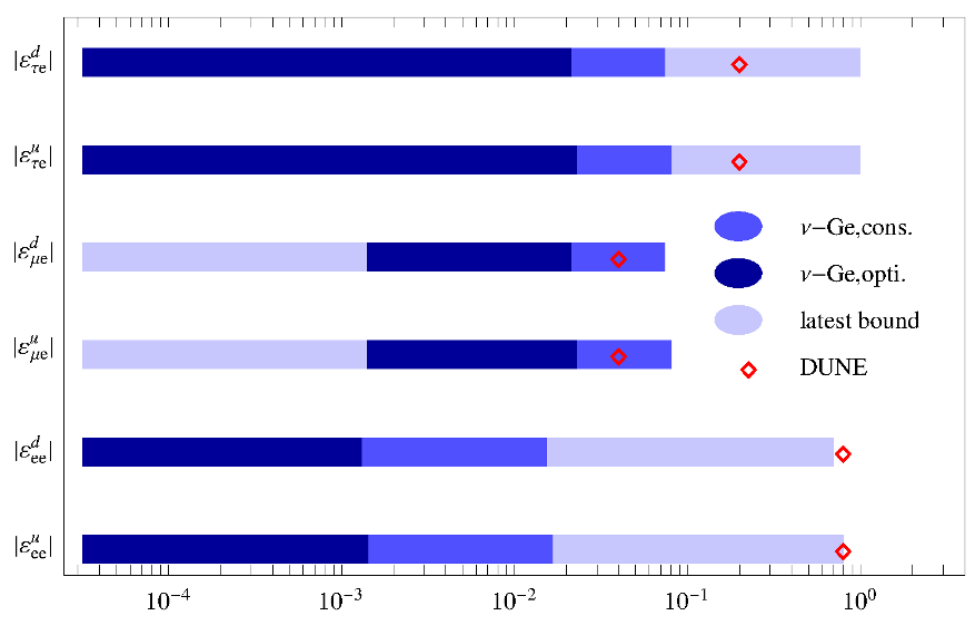

Figure 11. NSI sensitivities compared with the latest known bounds and the expected constraints from DUNE [43]. The label "latest bound" indicates the known constraints from ref. [16]. " $\nu$-Ge, opti." and " $\nu$-Ge, cons." are estimated sensitivities of our assumed $100 \mathrm{~kg}$ Ge detector running for 5 years with optimistic and conservative configurations, respectively.

\begin{tabular}{|ccccccc|}
\hline & $\varepsilon_{e e}^{u}$ & $\varepsilon_{e e}^{d}$ & $\varepsilon_{\mu e}^{u}$ & $\varepsilon_{\mu e}^{d}$ & $\varepsilon_{\tau e}^{u}$ & $\varepsilon_{\tau e}^{d}$ \\
\hline Conservative & $1.7 \times 10^{-2}$ & $1.5 \times 10^{-2}$ & $8.1 \times 10^{-2}$ & $7.5 \times 10^{-2}$ & $8.1 \times 10^{-2}$ & $7.5 \times 10^{-2}$ \\
\hline Intermediate & $6.0 \times 10^{-3}$ & $5.5 \times 10^{-3}$ & $4.8 \times 10^{-2}$ & $4.4 \times 10^{-2}$ & $4.8 \times 10^{-2}$ & $4.4 \times 10^{-2}$ \\
\hline Optimistic & $1.4 \times 10^{-3}$ & $1.3 \times 10^{-3}$ & $2.3 \times 10^{-2}$ & $2.1 \times 10^{-2}$ & $2.3 \times 10^{-2}$ & $2.1 \times 10^{-2}$ \\
\hline Latest bound [16] & 0.8 & 0.7 & $1.4 \times 10^{-3}$ & $1.4 \times 10^{-3}$ & 1.0 & 1.0 \\
\hline DUNE [43] & 0.8 & 0.8 & 0.04 & 0.04 & 0.2 & 0.2 \\
\hline
\end{tabular}

Table 1. $3 \sigma$-bounds on NSI parameters.

non-zero $R$ would stem from the interference of scalar and tensor interactions, i.e., would require the coexistence of two new interactions. Therefore we only consider three cases, $\left(\xi_{S}, \xi_{V}\right),\left(\xi_{A}, \xi_{V}\right)$, and $\left(\xi_{T}, \xi_{V}\right)$.

The result is given in figure 12 with both the conservative (left panels) and optimistic (right panels) configurations taken. In the conservative configuration, the sensitivity on $\xi_{V}$ is correlated with the other parameters. For example, if $\xi_{T}=0$ then $\xi_{V}$ would be only allowed to stay in the regime $0.88<\xi_{V}<1.06$ at $99.7 \%$ confidence level; if there is a sizable contribution from the tensor interaction with, say, $\xi_{T}=0.42$ then $\xi_{V}$ is allowed to significantly deviate from the SM value, going down to 0.68 . The correlation could be avoided if the systematic uncertainties and the threshold are improved to the optimistic configuration, as is shown in the right panels of figure 12. The qualitative explanation is that for large systematic uncertainties, the sensitivity will mainly depend on the total event number while the constraint from the spectrum information is not significant. In this case the tensor interaction will mimic the vector interaction in the signal, since they both contribute to the total event number. If the systematic uncertainties are small enough so that the spectrum is also measured to good accuracy, then the spectrum information could 

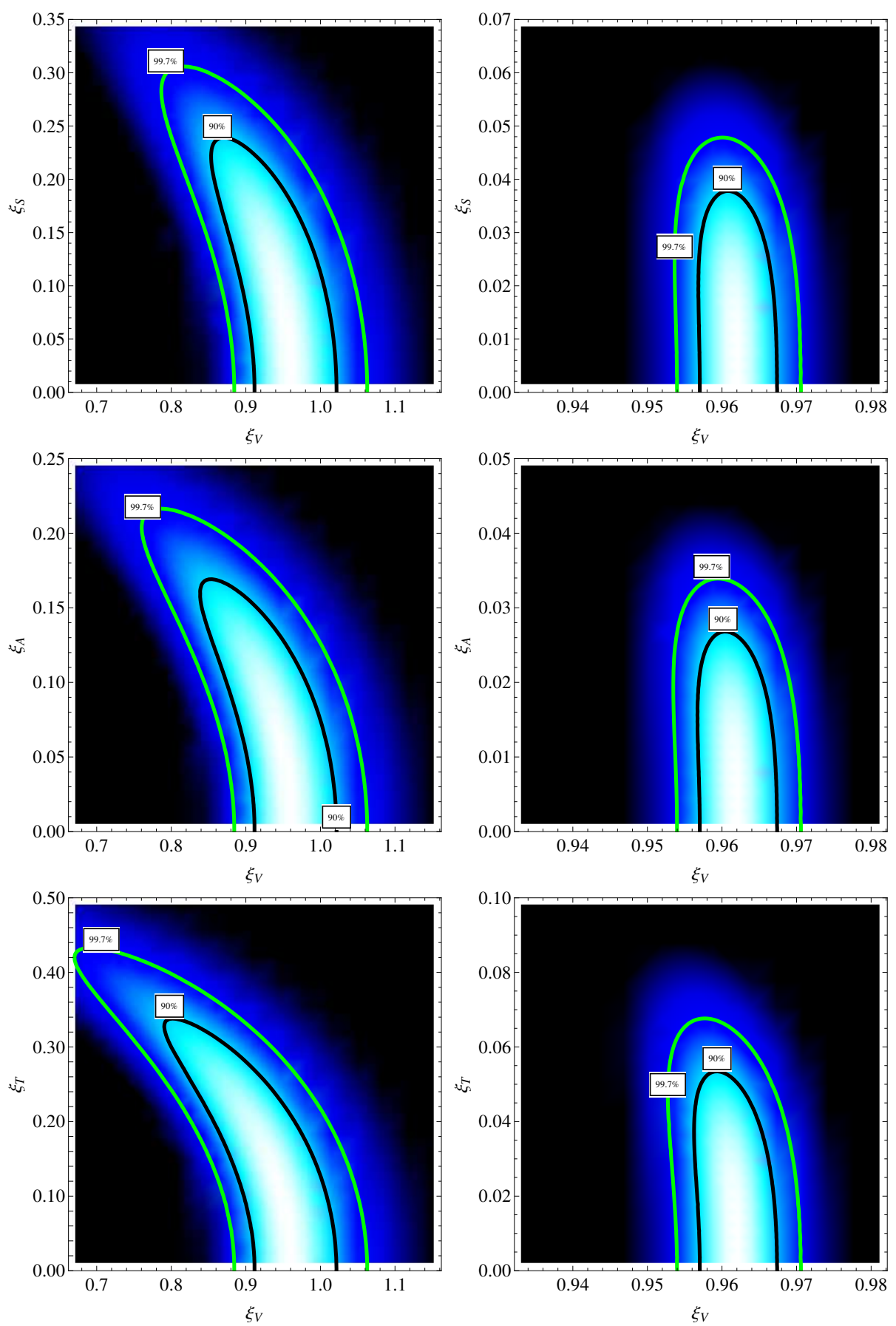

Figure 12. Sensitivity on the exotic neutral currents. The green and black contours correspond to $99.7 \%$ and $90 \%$ exclusion bounds. Left (right) panels are generated under the conservative (optimistic) configuration. 


\begin{tabular}{|ccccc|}
\hline & $\xi_{S}$ & $\xi_{V}$ & $\xi_{A}$ & $\xi_{T}$ \\
\hline Conservative & 0.21 & $(0.893,1.048)$ & 0.14 & 0.25 \\
\hline Intermediate & 0.11 & $(0.934,0.993)$ & $7.8 \times 10^{-2}$ & 0.14 \\
\hline Optimistic & $4.4 \times 10^{-2}$ & $(0.955,0.970)$ & $3.1 \times 10^{-2}$ & $5.9 \times 10^{-2}$ \\
\hline
\end{tabular}

Table 2. $3 \sigma$-bounds on exotic neutral current parameters, see eq. (4.9). The SM value of $\xi_{V}$ is 0.962 .

distinguish the contribution of the tensor interaction from the vector interaction. The same argument also applies for the other two cases $\left(\xi_{S}, \xi_{V}\right)$ and $\left(\xi_{A}, \xi_{V}\right)$. Therefore in future $\mathrm{C} \nu N \mathrm{~S}$ experiments reducing the systematic uncertainties is very important to distinguish signals from new exotic interactions and the SM interaction. The limits obtainable in our benchmark experiment are summarized in table 2 .

\section{Conclusion}

Future coherent neutrino-nucleus scattering experiments will provide exciting new data to test Standard Model and new neutrino physics to unprecedented accuracy. We have assumed here a future experiment with a low threshold (down to $0.1 \mathrm{keV}$ nuclear recoil) Germanium detector, with experimental benchmark numbers of $500 \mathrm{~kg} \times$ years $\times \mathrm{GW}$ reactor neutrinos and a baseline of $10 \mathrm{~m}$. We firmly believe that such a setup is achievable within the next decade, and it will provide event numbers of the order of $10^{5}$. Constraints on neutrino non-standard interactions and exotic neutral current interactions were evaluated. The expected sensitivities were shown to reach percent and permille level when compared to Fermi interaction, significantly better than expected constraints from oscillation experiments. We have demonstrated that such comparably compact coherent scattering experiments open a new window into exciting physics and should be pursued actively.

\section{Acknowledgments}

We thank Carlos Yaguna and Thomas Rink for many useful discussions. WR is supported by the DFG with grant RO 2516/6-1 in the Heisenberg Programme.

\section{A Cross section calculation of coherent $\nu-N$ scattering in the Standard Model}

In the $\mathrm{SM}$, the neutral current (NC) is

$$
\begin{aligned}
J_{\mathrm{NC}}^{\mu} & =2 \sum_{f} g_{L}^{f} \overline{f_{L}} \gamma^{\mu} f_{L}+g_{R}^{f} \overline{f_{R}} \gamma^{\mu} f_{R} \\
& =\sum_{f} \bar{f} \gamma^{\mu}\left(g_{V}^{f}-g_{A}^{f} \gamma^{5}\right) f,
\end{aligned}
$$


where $f$ stands for all elementary fermions in the SM and $f_{L, R}$ are their left/right-handed components,

$$
f_{L}=\frac{1-\gamma^{5}}{2} f, f_{R}=\frac{1+\gamma^{5}}{2} f .
$$

Here $g_{L, R}^{f}$ are determined by the quantum numbers of the corresponding fermions under $\mathrm{SU}(2)_{L} \times \mathrm{U}(1)_{Y}$ :

$$
\begin{aligned}
& g_{L}^{\nu}=\frac{1}{2}, \quad g_{R}^{\nu}=0, \quad g_{L}^{e}=-\frac{1}{2}+s_{W}^{2}, \quad g_{R}^{e}=s_{W}^{2} . \\
& g_{L}^{u}=\frac{1}{2}-\frac{2}{3} s_{W}^{2}, \quad g_{R}^{u}=-\frac{2}{3} s_{W}^{2}, \quad g_{L}^{d}=-\frac{1}{2}+\frac{1}{3} s_{W}^{2}, \quad g_{R}^{d}=\frac{1}{3} s_{W}^{2} .
\end{aligned}
$$

The vector/axial couplings $g_{V, A}^{f}$ are defined as

$$
g_{V}^{f}=g_{L}^{f}+g_{R}^{f}, g_{A}^{f}=g_{L}^{f}-g_{R}^{f} .
$$

At low energies the effective $\mathrm{NC}$ interaction is

$$
\mathcal{L}_{\mathrm{NC}}=\frac{G_{F}}{\sqrt{2}} J_{\mathrm{NC}}^{\mu} J_{\mathrm{NC} \mu}
$$

therefore the amplitude of the coherent $\nu-N$ scattering is

$$
i \mathcal{M}(\nu+N \rightarrow \nu+N)=-i \sqrt{2} G_{F}\left\langle N\left(k_{2}\right)\left|J_{\mathrm{NC}}^{\mu}\right| N\left(p_{2}\right)\right\rangle\left\langle\nu\left(k_{1}\right)\left|J_{\mathrm{NC} \mu}\right| \nu\left(p_{1}\right)\right\rangle,
$$

where $p_{1}, k_{1}, p_{2}, k_{2}$ are the momenta of the initial neutrino, final neutrino, initial nucleus and final nucleus, respectively.

The matrix element $\left\langle N\left(k_{2}\right)\left|J_{\mathrm{NC}}^{\mu}\right| N\left(p_{2}\right)\right\rangle$ only depends on the quark sector in $J_{\mathrm{NC}}^{\mu}$ since the nucleus is a bound state of many $u$ and $d$ quarks. So we have

$$
\begin{aligned}
\left\langle N\left|J_{\mathrm{NC}}^{\mu}\right| N\right\rangle= & g_{L}^{u}\left\langle N\left|\overline{u_{L}} \gamma^{\mu} u_{L}\right| N\right\rangle+g_{R}^{u}\left\langle N\left|\overline{u_{R}} \gamma^{\mu} u_{R}\right| N\right\rangle \\
& +g_{L}^{d}\left\langle N\left|\overline{d_{L}} \gamma^{\mu} d_{L}\right| N\right\rangle+g_{R}^{d}\left\langle N\left|\overline{d_{R}} \gamma^{\mu} d_{R}\right| N\right\rangle .
\end{aligned}
$$

Assuming that the nucleus does not violate parity, we have

$$
\left\langle N\left|\overline{u_{L}} \gamma^{\mu} u_{L}\right| N\right\rangle=\left\langle N\left|\overline{u_{R}} \gamma^{\mu} u_{R}\right| N\right\rangle,\left\langle N\left|\overline{d_{L}} \gamma^{\mu} d_{L}\right| N\right\rangle=\left\langle N\left|\overline{d_{R}} \gamma^{\mu} d_{R}\right| N\right\rangle
$$

Note that generally $|N\rangle$ does not have to respect parity symmetry. For example, if the whole nucleus is a spin- $1 / 2$ fermion then it is impossible for $|N\rangle$ to be invariant under the parity transformation which would flip the orientation of the spin. Even if the nucleus is a spin-0 particle, for the $u$ quarks the number of spin-up could be different from the number of spin-down. ${ }^{6}$ However, for a nucleus with a large mass number $A$, it contains many $u$ and $d$ quarks so that statistically we expect that they form a large object (the nucleus) that approximately respects parity.

\footnotetext{
${ }^{6}$ Besides, the distribution of protons and neutrons in a nucleus is not spherical, though it tends to be more spherical with increasing atomic number.
} 
Another relation we will use is

$$
\frac{\left\langle N\left|\bar{u} \gamma^{\mu} u\right| N\right\rangle}{\left\langle N\left|\bar{d} \gamma^{\mu} d\right| N\right\rangle}=\frac{2 Z+N}{2 N+Z}
$$

where $N$ is the number of neutrons and $Z$ the number of protons in the nucleus. Note that in a nucleus with $N$ neutrons and $Z$ protons, the numbers of $u$ and $d$ quarks are $2 Z+N$ and $2 N+Z$ respectively. Their ratio must be identical to the ratio of the above matrix elements if all the quarks are free particles. Since the strong interaction can not distinguish $u$ quarks and $d$ quarks, we assume that this relation holds for the bound quarks in the nucleus as well.

From eq. (A.11) we can write down

$$
\left\langle N\left|\bar{u} \gamma^{\mu} u\right| N\right\rangle=(2 Z+N) f^{\mu},\left\langle N\left|\bar{d} \gamma^{\mu} d\right| N\right\rangle=(2 N+Z) f^{\mu},
$$

where $f^{\mu}$ can be determined by the electromagnetic property of the nucleus. Let us first consider the electromagnetic current

$$
J_{\mathrm{EM}}^{\mu}=\frac{2}{3} \bar{u} \gamma^{\mu} u+\frac{-1}{3} \bar{d} \gamma^{\mu} d .
$$

From the Feynman rules of a complex scalar field with a gauged U(1) symmetry we know that the interaction vertex of the gauge boson with the scalar field should be proportional to $\left(p_{2}+k_{2}\right)^{\mu}$. Therefore we have

$$
\left\langle N\left(k_{2}\right)\left|J_{\mathrm{EM}}^{\mu}\right| N\left(p_{2}\right)\right\rangle=\left(p_{2}+k_{2}\right)^{\mu} Q_{\mathrm{nucl}} F\left(q^{2}\right),
$$

where $Q_{\text {nucl }}=Z$ is the electric charge of the nucleus and $q^{\mu}$ is the momentum transfer, defined as $q^{\mu} \equiv k_{2}^{\mu}-p_{2}^{\mu}$. From eqs. (A.12), (A.13) and (A.14), we obtain the form of $f^{\mu}$ :

$$
f^{\mu}=\left(p_{2}+k_{2}\right)^{\mu} F\left(q^{2}\right) .
$$

For very soft photons $\left(q^{2} \rightarrow 0\right)$ in the electromagnetic interaction, the nucleus radius $r_{\text {nucl }}$ is much smaller than the electromagnetic wavelength so that it can be treated as a point-like particle, with electric charge $Q_{\text {nucl }}$. Therefore for a very soft momentum transfer we have

$$
F\left(q^{2} \ll 1 / r_{\text {nucl }}^{2}\right) \approx 1 .
$$

With eqs. (A.10), (A.12) and (A.15), eq. (A.9) can now be written as

$$
\begin{aligned}
\left\langle N\left(k_{2}\right)\left|J_{\mathrm{NC}}^{\mu}\right| N\left(p_{2}\right)\right\rangle & =F\left(q^{2}\right)\left(p_{2}+k_{2}\right)^{\mu}\left[(2 Z+N) g_{V}^{u}+(2 N+Z) g_{V}^{d}\right] \\
& =F\left(q^{2}\right)\left(p_{2}+k_{2}\right)^{\mu}\left[Z g_{V}^{p}+N g_{V}^{n}\right]
\end{aligned}
$$

where

$$
g_{V}^{p}=\frac{1}{2}-2 s_{W}^{2} \quad g_{V}^{n}=-\frac{1}{2} .
$$

Some references $[9,15]$ define the weak charge $Q_{W}$ which is

$$
Q_{W}=-2\left(Z g_{V}^{p}+N g_{V}^{n}\right)=N-\left(1-4 s_{W}^{2}\right) Z .
$$


Now we can continue the evaluation of eq. (A.8)

$$
i \mathcal{M}^{s s^{\prime}}(\nu+N \rightarrow \nu+N)=i \frac{\sqrt{2}}{2} G_{F} Q_{W} F\left(q^{2}\right) g_{L}^{\nu}\left(p_{2}+k_{2}\right)^{\mu} \bar{v}^{s}\left(p_{1}\right) \gamma^{\mu}\left(1-\gamma^{5}\right) v^{s^{\prime}}\left(k_{1}\right),
$$

where $s$ and $s^{\prime}$ are the helicities of the initial neutrino and final neutrino, both left-handed. When computing $|i \mathcal{M}|^{2}$ we can also use the trace technology since the right-handed case should vanish due to the $V-A$ coupling of neutrinos in eq. (A.20),

$$
|i \mathcal{M}|^{2}=\sum_{s s^{\prime}}\left|i \mathcal{M}^{s s^{\prime}}\right|^{2}
$$

One can evaluate it immediately: ${ }^{7}$

$$
|i \mathcal{M}|^{2}=32 G_{F}^{2} Q_{W}^{2} F^{2}\left(g_{L}^{\nu}\right)^{2} M^{2} E_{\nu}^{2}\left(1-\frac{T}{E_{\nu}}-\frac{M T}{2 E_{\nu}^{2}}\right),
$$

where $M$ is the nucleus mass and $E_{\nu}$ the neutrino energy; $T$ is the recoil energy of the nucleus, which can be related to $c_{\theta} \equiv \cos \theta$, where $\theta$ is defined as the scattering angle between the momenta of the initial neutrino and final nucleus,

$$
T=\frac{2 M E_{\nu}^{2} c_{\theta}^{2}}{\left(M+E_{\nu}\right)^{2}-E_{\nu}^{2} c_{\theta}^{2}} .
$$

For a given value of $E_{\nu}$, the maximal recoil energy $T_{\max }$ is reached at $\theta=0$ :

$$
T_{\max }\left(E_{\nu}\right)=\frac{2 E_{\nu}^{2}}{M+2 E_{\nu}} .
$$

In the form factor $F\left(q^{2}\right), q^{2}$ is needed, which can be expressed in terms of $T$ as $q^{2}=-2 M T$.

The differential cross section in the laboratory frame is

$$
\frac{d \sigma}{d c_{\theta}}=\frac{|\mathcal{M}|^{2}}{8 \pi} \frac{c_{\theta}\left(E_{\nu}+M\right)^{2}}{\left[\left(M+E_{\nu}\right)^{2}-E_{\nu}^{2} c_{\theta}^{2}\right]^{2}},
$$

or

$$
\frac{d \sigma}{d T}=\frac{|\mathcal{M}|^{2}}{32 \pi M E_{\nu}^{2}} .
$$

With the result in eq. (A.22) we have

$$
\frac{d \sigma}{d T}=\frac{G_{F}^{2}\left(2 g_{L}^{\nu} Q_{W}\right)^{2} F^{2}\left(q^{2}\right)}{4 \pi} M\left(1-\frac{T}{E_{\nu}}-\frac{M T}{2 E_{\nu}^{2}}\right) .
$$

We have finally arrived at the expression of the SM cross section in eqs. (2.1)-(2.3).

Finally, considering that $E_{\nu} \ll M$, many expressions can be simplified under this approximation. From eqs. (A.23) and (A.24) we have

$$
T \approx T_{\max } c_{\theta}^{2}
$$

and

which gives

$$
1-\frac{T}{E_{\nu}}-\frac{M T}{2 E_{\nu}^{2}} \approx \sin ^{2} \theta+\mathcal{O}\left(\frac{E_{\nu}^{2}}{M^{2}}\right)
$$

$$
\frac{d \sigma}{d T} \approx \frac{\sigma_{0}^{\mathrm{SM}}}{M} \sin ^{2} \theta
$$

\footnotetext{
${ }^{7}$ Some kinetic relations are needed in the calculation, including $q^{2}=2 M T$ and $p_{1} \cdot q=-p_{2} \cdot q=q^{2} / 2$. The former is from $p_{2} \cdot k_{2}=M(M+T)$ and the latter is from the on-shell conditions of $k_{1}$ and $k_{2}$.
} 


\section{B What if $N$ is a spin-1/2 or spin-1 particle?}

We may ask whether non-zero spins have a significant effect on the calculation presented above or not. An intuitive estimation is that it should be only a weak effect. The reason is that a large nucleus contains many spin- $1 / 2$ fermions, i.e. protons and neutrons. They form the nucleus in which the proton and neutron spins almost cancel. If one proton flips its spin, the nucleus spin would be changed e.g. from 0 to 1 . Since we expect that the coherent $\nu-N$ scattering is insensitive to the status of a single proton inside the nucleus, we suspect that there should be no significant difference between zero and non-zero spins, as long as the non-zero spin is not very high.

For a spin-1/2 nucleus, eq. (A.17) is modified to

$$
\left\langle N\left(k_{2}, r^{\prime}\right)\left|J_{\mathrm{NC}}^{\mu}\right| N\left(p_{2}, r\right)\right\rangle=F\left(q^{2}\right) \bar{u}^{r^{\prime}}\left(k_{2}\right) \gamma^{\mu} u^{r}\left(p_{2}\right)\left[Z g_{V}^{p}+N g_{V}^{n}\right],
$$

where $\bar{u}^{r^{\prime}}\left(k_{2}\right)$ and $\bar{u}^{r}\left(p_{2}\right)$ denote the finial and initial states of the Dirac particle, i.e., the spin- $1 / 2$ nucleus. Then eq. (A.20) is changed to

$i \mathcal{M}^{r^{\prime} r s s^{\prime}}(\nu+N \rightarrow \nu+N)=i \frac{\sqrt{2}}{2} G_{F} Q_{W} F\left(q^{2}\right) g_{L}^{\nu}\left[\bar{u}^{r^{\prime}}\left(k_{2}\right) \gamma^{\mu} u^{r}\left(p_{2}\right)\right]\left[\bar{u}^{s^{\prime}}\left(k_{1}\right) \gamma^{\mu}\left(1-\gamma^{5}\right) u^{s}\left(p_{1}\right)\right]$.

The above amplitude is for neutrinos while for antineutrinos it should be

$i \mathcal{M}^{r^{\prime} r s s^{\prime}}(\bar{\nu}+N \rightarrow \bar{\nu}+N)=i \frac{\sqrt{2}}{2} G_{F} Q_{W} F\left(q^{2}\right) g_{L}^{\nu}\left[\bar{u}^{r^{\prime}}\left(k_{2}\right) \gamma^{\mu} u^{r}\left(p_{2}\right)\right]\left[\bar{v}^{s}\left(p_{1}\right) \gamma^{\mu}\left(1-\gamma^{5}\right) v^{s^{\prime}}\left(k_{1}\right)\right]$.

Eq. (B.2) and eq. (B.3) essentially give the same $|\mathcal{M}|^{2}$ and thus the same cross section, as one can check by direct computation. The reason is due to the assumption that the interaction of the nucleus with the $Z$ boson is parity-conserved. If there is axial current in eq. (B.1), i.e., a $\gamma^{\mu} \gamma^{5}$ between $\bar{u}^{r^{\prime}}\left(k_{2}\right)$ and $u^{r}\left(p_{2}\right)$ then the $\nu N$ and $\bar{\nu} N$ cross sections would be different.

After evaluating the traces of the Dirac matrices in the amplitude, we get

$$
|\mathcal{M}|^{2}=\sum_{s s^{\prime}} \frac{1}{2} \sum_{r r^{\prime}}\left|i \mathcal{M}^{r^{\prime} r s s^{\prime}}\right|^{2}=32 G_{F}^{2} Q_{W}^{2} F^{2}\left(g_{L}^{\nu}\right)^{2} M^{2} E_{\nu}^{2}\left(1-\frac{T}{E_{\nu}}-\frac{M T}{2 E_{\nu}^{2}}+\frac{T^{2}}{2 E_{\nu}^{2}}\right),
$$

and then

$$
\left.\frac{d \sigma}{d T}\right|_{\text {spin- } 1 / 2}=\frac{G_{F}^{2}\left(2 g_{L}^{\nu} Q_{W}\right)^{2} F^{2}\left(q^{2}\right)}{4 \pi} M\left(1-\frac{T}{E_{\nu}}-\frac{M T}{2 E_{\nu}^{2}}+\frac{T^{2}}{2 E_{\nu}^{2}}\right) .
$$

This is the result for a spin-0 nucleus plus small negligible corrections, see eq. (2.4).

\section{Relations of $\left(C_{a}, \bar{D}_{a}\right)$ with $\left(C_{a}^{(q)}, \bar{D}_{a}^{(q)}\right)$}

In section 4 when discussing exotic neutral currents we defined the nucleus couplings $\left(C_{a}, \bar{D}_{a}\right)$ and the quark couplings $\left(C_{a}^{(q)}, \bar{D}_{a}^{(q)}\right)$. In this appendix we will derive the relations of $\left(C_{a}, \bar{D}_{a}\right)$ to $\left(C_{a}^{(q)}, \bar{D}_{a}^{(q)}\right)$ by comparing the scattering amplitudes. 
Starting from the fundamental Lagrangian (4.1), we can write down the amplitude

$$
i \mathcal{M}^{s^{\prime} s r^{\prime} r}=-i \frac{G_{F}}{\sqrt{2}} \bar{v}^{s^{\prime}}\left(p_{1}\right) P_{R} \Gamma^{a} v^{s}\left(k_{1}\right)\left\langle\Gamma^{a}\right\rangle_{N}^{r^{\prime} r}
$$

where

$$
\left\langle\Gamma^{a}\right\rangle_{N}^{r^{\prime} r} \equiv\left\langle N\left(k_{2}, r^{\prime}\right)\left|\sum_{q=u, d} \bar{q} \Gamma^{a}\left(C_{a}^{(q)}+\bar{D}_{a}^{(q)} i \gamma^{5}\right) q\right| N\left(p_{2}, r\right)\right\rangle
$$

To compute the amplitude we need to know $\left\langle N\left|\bar{q} \Gamma^{a} q\right| N\right\rangle$. Similar to eq. (A.11), here we also assume that

$$
\frac{\left\langle N\left|\bar{u} \Gamma^{a} u\right| N\right\rangle}{\left\langle N\left|\bar{d} \Gamma^{a} d\right| N\right\rangle}=\frac{n_{u}}{n_{d}}
$$

which enables us to define

$$
F^{a} \equiv \frac{\left\langle N\left|\bar{u} \Gamma^{a} u\right| N\right\rangle}{n_{u}}=\frac{\left\langle N\left|\bar{d} \Gamma^{a} d\right| N\right\rangle}{n_{d}} .
$$

For instance, generalizing eq. (B.1), a scalar interaction of down quarks $\langle N|\bar{d} d| N\rangle$, can result in a term $\bar{u} u$ or $\bar{u} \gamma_{5} u$, where $u$ is a Dirac spinor. Both terms come with a form factor, and we have neglected terms involving momenta. This implies that

$$
F^{S}=f_{S S} \bar{u}^{r^{\prime}}\left(k_{2}\right) \Gamma^{S} u^{r}\left(p_{2}\right)+f_{S P} \bar{u}^{r^{\prime}}\left(k_{2}\right) \Gamma^{P} u^{r}\left(p_{2}\right) .
$$

In analogy, we can write the other terms as

$$
\begin{aligned}
& F^{P}=f_{P S} \bar{u}^{r^{\prime}}\left(k_{2}\right) \Gamma^{P} u^{r}\left(p_{2}\right)+f_{P P} \bar{u}^{r^{\prime}}\left(k_{2}\right) \Gamma^{S} u^{r}\left(p_{2}\right), \\
& F^{V}=f_{V V} \bar{u}^{r^{\prime}}\left(k_{2}\right) \Gamma^{V} u^{r}\left(p_{2}\right)+f_{V A} \bar{u}^{r^{\prime}}\left(k_{2}\right) \Gamma^{A} u^{r}\left(p_{2}\right), \\
& F^{A}=f_{A V} \bar{u}^{r^{\prime}}\left(k_{2}\right) \Gamma^{A} u^{r}\left(p_{2}\right)+f_{A A} \bar{u}^{r^{\prime}}\left(k_{2}\right) \Gamma^{V} u^{r}\left(p_{2}\right), \\
& F^{T}=f_{T} \bar{u}^{r^{\prime}}\left(k_{2}\right) \Gamma^{T} u^{r}\left(p_{2}\right)+f_{T^{\prime}} \bar{u}^{r^{\prime}}\left(k_{2}\right) \Gamma^{T}\left(i \gamma^{5}\right) u^{r}\left(p_{2}\right),
\end{aligned}
$$

where all the $f$ are form factors. We will not address the calculation of form factors in this paper, see refs. $[44,45]$ and references therein.

From the definition (C.4), we can express $\left\langle\Gamma^{a}\right\rangle_{N}$ in terms of $F^{a}$ :

$$
\begin{aligned}
\left\langle\Gamma^{S}\right\rangle_{N} & =\sum_{q=u, d} n_{q}\left(C_{S}^{(q)} F^{S}+\bar{D}_{S}^{(q)} F^{P}\right) \\
\left\langle\Gamma^{P}\right\rangle_{N} & =\sum_{q=u, d} n_{q}\left(C_{P}^{(q)} F^{P}-\bar{D}_{P}^{(q)} F^{S}\right) \\
\left\langle\Gamma^{V}\right\rangle_{N} & =\sum_{q=u, d} n_{q}\left(C_{V}^{(q)} F^{V}+i \bar{D}_{V}^{(q)} F^{A}\right) \\
\left\langle\Gamma^{A}\right\rangle_{N} & =\sum_{q=u, d} n_{q}\left(C_{A}^{(q)} F^{A}+i \bar{D}_{A}^{(q)} F^{V}\right) \\
\left\langle\Gamma^{T}\right\rangle_{N}^{\mu \nu} & =\sum_{q=u, d} n_{q}\left[C_{T}^{(q)}\left(F^{T}\right)^{\mu \nu}-\frac{1}{2} \epsilon^{\mu \nu \rho \sigma} \bar{D}_{T}^{(q)}\left(F^{T}\right)_{\rho \sigma}\right] .
\end{aligned}
$$


We have suppressed the spin indices $\left(r, r^{\prime}, \ldots\right)$ and Lorentz indices $(\mu, \nu, \ldots)$ in the above relations except for eq. (C.14) where we need the Lorentz indices to explicitly express the relation.

By writing the amplitude (C.1) in terms of $\left(C_{a}^{(q)}, \bar{D}_{a}^{(q)}\right)$ and the form factors, and comparing it with eq. (4.7), we obtain

$$
\begin{aligned}
\left(C_{S}, \bar{D}_{S}\right) & =\sum_{q=u, d} n_{q}\left(C_{S}^{(q)} f_{S S}+\bar{D}_{S}^{(q)} f_{P P}, C_{S}^{(q)} f_{S P}+\bar{D}_{S}^{(q)} f_{P S}\right), \\
\left(C_{P}, \bar{D}_{P}\right) & =\sum_{q=u, d} n_{q}\left(C_{P}^{(q)} f_{P S}-\bar{D}_{S}^{(q)} f_{S P},-C_{P}^{(q)} f_{P P}+\bar{D}_{P}^{(q)} f_{S S}\right), \\
\left(C_{V}, i \bar{D}_{V}\right) & =\sum_{q=u, d} n_{q}\left(C_{V}^{(q)} f_{V V}+i \bar{D}_{V}^{(q)} f_{A A}, C_{V}^{(q)} f_{V A}+i \bar{D}_{V}^{(q)} f_{A V}\right), \\
\left(C_{A}, i \bar{D}_{A}\right) & =\sum_{q=u, d} n_{q}\left(C_{A}^{(q)} f_{A V}+i \bar{D}_{A}^{(q)} f_{V A}, C_{A}^{(q)} f_{A A}+i \bar{D}_{A}^{(q)} f_{V V}\right), \\
\left(C_{T}, \bar{D}_{T}\right) & =\sum_{q=u, d} n_{q}\left(C_{T}^{(q)} f_{T}-\bar{D}_{T}^{(q)} f_{T^{\prime}}, C_{T}^{(q)} f_{T^{\prime}}+\bar{D}_{T}^{(q)} f_{T}\right) .
\end{aligned}
$$

These are the relations that connect the nucleus couplings $\left(C_{a}, \bar{D}_{a}\right)$ and the quark couplings $\left(C_{a}^{(q)}, \bar{D}_{a}^{(q)}\right)$.

Open Access. This article is distributed under the terms of the Creative Commons Attribution License (CC-BY 4.0), which permits any use, distribution and reproduction in any medium, provided the original author(s) and source are credited.

\section{References}

[1] D.Z. Freedman, Coherent neutrino nucleus scattering as a probe of the weak neutral current, Phys. Rev. D 9 (1974) 1389 [inSPIRE].

[2] D.Z. Freedman, D.N. Schramm and D.L. Tubbs, The Weak Neutral Current and Its Effects in Stellar Collapse, Ann. Rev. Nucl. Part. Sci. 27 (1977) 167 [inSPIRE].

[3] A. Drukier and L. Stodolsky, Principles and Applications of a Neutral Current Detector for Neutrino Physics and Astronomy, Phys. Rev. D 30 (1984) 2295 [InSPIRE].

[4] J. Billard, L. Strigari and E. Figueroa-Feliciano, Implication of neutrino backgrounds on the reach of next generation dark matter direct detection experiments, Phys. Rev. D 89 (2014) 023524 [arXiv: 1307.5458] [INSPIRE].

[5] J. Barranco, O.G. Miranda and T.I. Rashba, Probing new physics with coherent neutrino scattering off nuclei, JHEP 12 (2005) 021 [hep-ph/0508299] [INSPIRE].

[6] B. Dutta, R. Mahapatra, L.E. Strigari and J.W. Walker, Sensitivity to Z-prime and nonstandard neutrino interactions from ultralow threshold neutrino-nucleus coherent scattering, Phys. Rev. D 93 (2016) 013015 [arXiv: 1508.07981] [INSPIRE].

[7] B. Dutta, Y. Gao, R. Mahapatra, N. Mirabolfathi, L.E. Strigari and J.W. Walker, Sensitivity to oscillation with a sterile fourth generation neutrino from ultra-low threshold neutrino-nucleus coherent scattering, Phys. Rev. D 94 (2016) 093002 [arXiv:1511.02834] [INSPIRE]. 
[8] D.K. Papoulias and T.S. Kosmas, Standard and Nonstandard Neutrino-Nucleus Reactions Cross sections and Event Rates to Neutrino Detection Experiments, Adv. High Energy Phys. 2015 (2015) 763648 [arXiv:1502.02928] [INSPIRE].

[9] K. Scholberg, Prospects for measuring coherent neutrino-nucleus elastic scattering at a stopped-pion neutrino source, Phys. Rev. D 73 (2006) 033005 [hep-ex/0511042] [INSPIRE].

[10] H.T. Wong, H.-B. Li, J. Li, Q. Yue and Z.-Y. Zhou, Research program towards observation of neutrino-nucleus coherent scattering, J. Phys. Conf. Ser. 39 (2006) 266 [hep-ex/0511001] [INSPIRE].

[11] COHERENT collaboration, D. Akimov et al., The COHERENT Experiment at the Spallation Neutron Source, arXiv:1509.08702 [INSPIRE].

[12] H.T.-K. Wong, Taiwan EXperiment On NeutrinO, The Universe 3 (2015) 22 [arXiv: 1608.00306] [INSPIRE].

[13] TEXONO collaboration, S. Kerman et al., Coherency in Neutrino-Nucleus Elastic Scattering, Phys. Rev. D 93 (2016) 113006 [arXiv: 1603.08786] [INSPIRE].

[14] TEXONO collaboration, A.K. Soma et al., Characterization and Performance of Germanium Detectors with sub-keV Sensitivities for Neutrino and Dark Matter Experiments, Nucl. Instrum. Meth. A 836 (2016) 67 [arXiv:1411.4802] [INSPIRE].

[15] A.J. Anderson, J.M. Conrad, E. Figueroa-Feliciano, K. Scholberg and J. Spitz, Coherent Neutrino Scattering in Dark Matter Detectors, Phys. Rev. D 84 (2011) 013008 [arXiv: 1103.4894] [INSPIRE].

[16] S. Davidson, C. Pena-Garay, N. Rius and A. Santamaria, Present and future bounds on nonstandard neutrino interactions, JHEP 03 (2003) 011 [hep-ph/0302093] [INSPIRE].

[17] D.G. Cerdeño, M. Fairbairn, T. Jubb, P.A.N. Machado, A.C. Vincent and C. Bøhm, Physics from solar neutrinos in dark matter direct detection experiments, JHEP 05 (2016) 118 [Erratum ibid. 09 (2016) 048] [arXiv: 1604.01025] [INSPIRE].

[18] J. Erler and M.J. Ramsey-Musolf, The Weak mixing angle at low energies, Phys. Rev. D 72 (2005) 073003 [hep-ph/0409169] [INSPIRE].

[19] D. Barker and D.M. Mei, Germanium Detector Response to Nuclear Recoils in Searching for Dark Matter, Astropart. Phys. 38 (2012) 1 [arXiv:1203.4620] [INSPIRE].

[20] J. Lindhard et al., Range concepts and heavy ion ranges (notes on atomic collisions, II), Mat. Fys. Medd. K. Dan. Vidensk. Selsk. 33 (1963) 1.

[21] V.I. Kopeikin, Flux and spectrum of reactor antineutrinos, Phys. Atom. Nucl. 75 (2012) 143 [INSPIRE].

[22] A.G. Beda et al., Gemma experiment: The results of neutrino magnetic moment search, Phys. Part. Nucl. Lett. 10 (2013) 139.

[23] G. Heusser et al., GIOVE - A new detector setup for high sensitivity germanium spectroscopy at shallow depth, Eur. Phys. J. C 75 (2015) 531 [arXiv:1507.03319] [INSPIRE].

[24] B. Achkar et al., Comparison of anti-neutrino reactor spectrum models with the Bugey-3 measurements, Phys. Lett. B 374 (1996) 243 [INSPIRE].

[25] K. Schreckenbach, G. Colvin, W. Gelletly and F. Von Feilitzsch, Determination of the anti-neutrino spectrum from U-235 thermal neutron fission products up to 9.5-MeV, Phys. Lett. B 160 (1985) 325 [INSPIRE].

[26] G. Mention et al., The Reactor Antineutrino Anomaly, Phys. Rev. D 83 (2011) 073006 [arXiv:1101.2755] [INSPIRE]. 
[27] P. Huber, On the determination of anti-neutrino spectra from nuclear reactors, Phys. Rev. C 84 (2011) 024617 [Erratum ibid. C 85 (2012) 029901] [arXiv: 1106.0687] [INSPIRE].

[28] RENO collaboration, S.-H. Seo, New Results from RENO and The $5 \mathrm{MeV}$ Excess, AIP Conf. Proc. 1666 (2015) 080002 [arXiv: 1410.7987] [InSPIRE].

[29] RENO collaboration, J.H. Choi et al., Observation of Energy and Baseline Dependent Reactor Antineutrino Disappearance in the RENO Experiment, Phys. Rev. Lett. 116 (2016) 211801 [arXiv: 1511.05849] [INSPIRE].

[30] DaYa BAY collaboration, F.P. An et al., Measurement of the Reactor Antineutrino Flux and Spectrum at Daya Bay, Phys. Rev. Lett. 116 (2016) 061801 [arXiv:1508.04233] [INSPIRE].

[31] Double CHOOZ collaboration, Y. Abe et al., Measurement of $\theta_{13}$ in Double CHOOZ using neutron captures on hydrogen with novel background rejection techniques, JHEP 01 (2016) 163 [arXiv: 1510.08937] [INSPIRE].

[32] C. Buck, A.P. Collin, J. Haser and M. Lindner, Investigating the Spectral Anomaly with Different Reactor Antineutrino Experiments, Phys. Lett. B 765 (2017) 159 [arXiv: 1512.06656] [INSPIRE].

[33] C. Giunti, Precise determination of the ${ }^{235} \mathrm{U}$ reactor antineutrino cross section per fission, Phys. Lett. B 764 (2017) 145 [arXiv: 1608.04096] [inSPIRE].

[34] P. Huber, The $5 \mathrm{MeV}$ bump - a nuclear whodunit mystery, Phys. Rev. Lett. 118 (2017) 042502 [arXiv: 1609.03910] [INSPIRE].

[35] T. Ohlsson, Status of non-standard neutrino interactions, Rept. Prog. Phys. 76 (2013) 044201 [arXiv: 1209. 2710] [INSPIRE].

[36] J. Heeck and W. Rodejohann, Gauged $L_{\mu}-L_{\tau}$ and different Muon Neutrino and Anti-Neutrino Oscillations: MINOS and beyond, J. Phys. G 38 (2011) 085005 [arXiv: 1007.2655] [INSPIRE].

[37] CHARM collaboration, J. Dorenbosch et al., Experimental Verification of the Universality of $\nu_{e}$ and $\nu_{\mu}$ Coupling to the Neutral Weak Current, Phys. Lett. B 180 (1986) 303 [InSPIRE].

[38] A. de Gouvêa, S. Lola and K. Tobe, Lepton flavor violation in supersymmetric models with trilinear R-parity violation, Phys. Rev. D 63 (2001) 035004 [hep-ph/0008085] [INSPIRE].

[39] V. Shtabovenko, R. Mertig and F. Orellana, New Developments in FeynCalc 9.0, Comput. Phys. Commun. 207 (2016) 432 [arXiv: 1601.01167] [INSPIRE].

[40] R. Mertig, M. Böhm and A. Denner, FEYN CALC: Computer algebraic calculation of Feynman amplitudes, Comput. Phys. Commun. 64 (1991) 345 [InSPIRE].

[41] H.H. Patel, Package-X: A Mathematica package for the analytic calculation of one-loop integrals, Comput. Phys. Commun. 197 (2015) 276 [arXiv:1503.01469] [INSPIRE].

[42] N. Berger et al., Measuring the weak mixing angle with the P2 experiment at MESA, J. Univ. Sci. Tech. China 46 (2016) 481 [arXiv:1511.03934] [INSPIRE].

[43] A. de Gouvêa and K.J. Kelly, Non-standard Neutrino Interactions at DUNE, Nucl. Phys. B 908 (2016) 318 [arXiv:1511.05562] [INSPIRE].

[44] G. Bélanger, F. Boudjema, A. Pukhov and A. Semenov, Dark matter direct detection rate in a generic model with MicrOMEGAs 2.2, Comput. Phys. Commun. 180 (2009) 747 [arXiv: 0803.2360] [INSPIRE].

[45] F. Bishara, J. Brod, B. Grinstein and J. Zupan, Chiral Effective Theory of Dark Matter Direct Detection, JCAP 02 (2017) 009 [arXiv:1611.00368] [INSPIRE]. 